\title{
REDUCED MHD IN ASTROPHYSICAL APPLICATIONS: TWO-DIMENSIONAL OR THREE-DIMENSIONAL?
}

\author{
S. Oughton, ${ }^{1}$ W. H. Matthaeus, ${ }^{2}$ and P. DmitruK ${ }^{3}$ \\ ${ }^{1}$ Department of Mathematics and Statistics, University of Waikato, Hamilton NZ \\ ${ }^{2}$ Department of Physics and Astronomy, University of Delaware, Delaware 19716, USA \\ ${ }^{3}$ Departamento de Física, Facultad de Ciencias Exactas y Naturales, Universidad de Buenos Aires-Conicet, Argentina
}

\section{ABSTRACT}

Originally proposed as an efficient approach to computation of nonlinear dynamics in tokamak fusion research devices, Reduced Magnetohydrodynamics (RMHD) has subsequently found application in studies of coronal heating, flux tube dynamics, charged particle transport and, in general, as an approximation to describe plasma turbulence in space physics and astrophysics. Given the diverse set of derivations available in the literature, there has emerged some level of discussion and lack of consensus regarding the completeness of RMHD as a turbulence model, and its applicability in contexts such as the solar wind. Some of the key issues in this discussion are examined here, emphasizing that RMHD is properly neither $2 \mathrm{D}$ nor fully $3 \mathrm{D}$, being rather an incomplete representation that enforces at least one family of extraneous conservation laws.

\section{INTRODUCTION}

Reduced magnetohydrodynamics (RMHD) is an incompressible fluid model of plasma behavior that is simpler than a full MHD model. It thus has the advantage of being computationally more efficient for many problems for which it is applicable. It is therefore relevant to understand in some detail the circumstances in which RMHD may be derived as a suitable approximation to a full MHD model. Providing such clarification is the goal of the present paper.

The RMHD model involves a mean (often uniform) magnetic field $\boldsymbol{B}_{0}=B_{0} \hat{\boldsymbol{z}}$ that is necessarily energetically strong compared with the fluctuating magnetic field $\boldsymbol{b}$ and the fluctuating velocity field $\boldsymbol{v}$. In addition, the fluctuating quantities all vary more slowly along the mean field direction than in the transverse directions, so that the corresponding gradients satisfy $\left|\nabla_{\|}\right| \ll\left|\nabla_{\perp}\right|$. A third important feature is the absence of parallel fluctuations ${ }^{1}$ with $\boldsymbol{B}_{0} \cdot \boldsymbol{b}=0$ and $\boldsymbol{B}_{0} \cdot \boldsymbol{v}=0$. These conditions emerge during the derivation (see $\S 2$ ) of the RMHD equations, which may be written as

$$
\begin{aligned}
& \frac{\partial \boldsymbol{v}}{\partial t}+\boldsymbol{v} \cdot \nabla_{\perp} \boldsymbol{v}=\boldsymbol{b} \cdot \nabla_{\perp} \boldsymbol{b}+B_{0} \frac{\partial \boldsymbol{b}}{\partial z}-\nabla_{\perp} p^{*}+\nu \nabla_{\perp}^{2} \boldsymbol{v},(1) \\
& \frac{\partial \boldsymbol{b}}{\partial t}+\boldsymbol{v} \cdot \nabla_{\perp} \boldsymbol{b}=\boldsymbol{b} \cdot \nabla_{\perp} \boldsymbol{v}+B_{0} \frac{\partial \boldsymbol{v}}{\partial z}+\eta \nabla_{\perp}^{2} \boldsymbol{b} .
\end{aligned}
$$

Here $\nu$ and $\eta$ are uniform constant viscosity and resistivity, magnetic fields are written in Alfvén speed units, $\partial / \partial z$ is the derivative in the direction parallel to $\boldsymbol{B}_{0}$, and $p^{*}$ is the magnetic pressure plus the incompressible fluid pressure. These dynamical equations are supplemented by the constraints $\nabla_{\perp} \cdot \boldsymbol{b}=0$ and $\nabla_{\perp} \cdot \boldsymbol{v}=0$. As stated, the equations are appropriate for cartesian (Strauss 1976, hereafter S76) or periodic (Dahlburg et al. 1985) geometry, but can also be formulated in curvilinear coordinates (Kadomtsev \& Pogutse 1974, hereafter KP).

Although initially developed as a simplified model for the nonlinear evolution of tokamak plasmas with large aspect ratios, more recent derivations of RMHD have

${ }^{1}$ When the plasma beta is not large. See $\S 2.5$. attempted to place the approximations in a broader context. In these there is a clear emphasis on the issue of elimination of high-frequency motions, which is rather implicit in the original formulations (KP, S76). The view of RMHD as a slow manifold limit of MHD for the case of a strong guide field pervades the later derivations, such as Montgomery (1982, hereafter M82), Zank \& Matthaeus (1992, hereafter ZM92), and Kinney \& McWilliams (1998). A general outline of the slow timescale approach is given in $§ 2.3$.

Following on from its tokamak origins, RMHD has also been employed in a variety of space physics and astrophysics contexts, including coronal loop dynamics, coronal heating, solar wind acceleration, reconnection, and particle acceleration (e.g., Milano et al. 1999; Oughton et al. 2001; van Ballegooijen et al. 2011; Dahlburg et al. 1985; Rappazzo et al. 2008, 2010, 2013; Dalena et al. 2014). Investigations of the turbulence properties of RMHD have also been carried out (e.g., Dahlburg et al. 1985; Kinney \& McWilliams 1997; Dmitruk et al. 2003; Oughton et al. 2004; Rappazzo et al. 2010, 2012).

While it is manifestly clear that RMHD represents a "reduction" of the full MHD model, what is less clear, but still an important issue in applications, is the question of how much physics is retained in RMHD, and what sorts of physics is lost due to the simplifications inherent in its derivation. At issue are even basic questions of dimensionality: Does RMHD remain close to the conditions of two dimensionality, as assumed for example in some coronal heating studies (Dmitruk \& Gómez 1997; Einaudi et al. 1996)? Or in contrast, may it be argued that RMHD is a suitable substitute for the full 3D incompressible MHD equations (e.g., Perez \& Boldyrev 2008; Beresnyak 2012; Mason et al. 2012). Further with regard to this dichotomy, if three dimensional effects are an essential feature of a particular MHD problem, will an investigation in terms of RMHD reveal those effects? A sensible answer to these questions will doubtless conclude that RMHD is in some sense intermediate to two dimensions and three, and also that some three dimensional effects will be captured in RMHD and others, not.

The purpose of the present paper is to clarify in some 
Table 1

Similarities and differences between MHD in various dimensions and leading-order RMHD. Here, 2.5D means that the vector fields have three Cartesian components that depend on the transverse coordinates $x$ and $y$, but not on the parallel coordinate $z$. A $\checkmark$ means the property or feature can occur within that model, a '-' that it cannot or does not.

\begin{tabular}{|l|ccc|}
\hline Feature & $2 \mathrm{D} / 2.5 \mathrm{D}$ & $3 \mathrm{D}$ & RMHD \\
\hline out of plane $B_{0} \hat{\boldsymbol{z}}$ & $-/ \checkmark$ & $\checkmark$ & $\checkmark$ \\
$\|$ coordinate & ignorable & general & large-scale \\
$\|$ variances & $-/ \checkmark$ & $\checkmark$ & $-(a)$ \\
$\|$ spectral transfer & - & $\checkmark$ & weak \\
strong $\perp$ transfer & $\checkmark$ & $\checkmark$ & $\checkmark$ \\
low-freq A waves & only $\omega=0$ & $\checkmark$ & $\checkmark$ \\
high-freq A waves & - & $\checkmark$ & - \\
slab waves $\left(k_{\perp}=0\right)$ & - & $\checkmark$ & - \\
$\|$ dissipation & - & $\checkmark$ & - \\
$\|$ shocks & - & $\checkmark$ & - \\
antidynamo theorem & $\checkmark$ & - & - \\
$E, H_{c}$ conserved & $\checkmark$ & $\checkmark$ & $\checkmark$ \\
$\left\langle a_{z}^{2}\right\rangle$ conserved & $\checkmark$ & - & - \\
magnetic helicity $H_{m}$ & - & $\checkmark$ & $\left\langle a_{z} B_{0}\right\rangle^{(b)}$ \\
$1 / f$ noise & $\checkmark$ & strong $B_{0}$ & $\checkmark$ \\
wave eqn for $W^{ \pm}\left(s^{\prime}\right)$ & - & - & $\checkmark$ \\
\hline
\end{tabular}

(a) Parallel variances are possible for large $\beta_{\mathrm{p}}$ or if the original system is incompressible. See $\S 2.5$.

(b) If $B_{0}$ is uniform this becomes $B_{0}\left\langle a_{z}\right\rangle$.

detail these issues, making clear which properties RMHD has in common with either three dimensional, or two dimensional MHD, and which features of each are absent in RMHD. ${ }^{2}$ Moreover, RMHD can also be seen to have some peculiar properties present in neither strictly two dimensional models, nor in three dimensional models. Table 1 provides a 'quick reference' overview of some distinctions between RMHD and other models. More detailed discussion of these points is given in the remainder of the paper.

\section{REVIEW OF DERIVATIONS}

The persistence of questions regarding the nature and applicability of RMHD derives at least in part from the very different styles found in its several derivations. Sometimes called the Strauss equations, RMHD has been derived starting from ideal compressible 3D MHD (KP; S76). Alternative and/or more rigorous derivations followed (M82; ZM92; Kinney \& McWilliams 1998), including Hamiltonian formulations (Morrison \& Hazeltine 1984) and consideration as a fluid limit of gyrokinetics (Schekochihin et al. 2009). As these derivations are readily available we do not repeat them in detail here, but rather summarize several strategies that have been employed in these developments. We then turn to a more detailed description of the distillation of RMHD from full MHD using timescale arguments, which we believe represents the most physically based approach. Following this perspective on the approximations and assumptions associated with RMHD we will be in a position to discuss some of its properties in greater depth. We note that derivations of reduced equations that extend RMHD to a

\footnotetext{
2 Our discussion examines the relationship between RMHD and MHD at the fluid level. See, e.g., Schekochihin et al. (2009) for the status of RMHD in a kinetic perspective.
}

weakly inhomogeneous medium have also been developed (Bhattacharjee et al. 1998; Kruger et al. 1998; van Ballegooijen et al. 2011), although these will not be discussed herein.

\subsection{Issues and parameters}

RMHD involves several interrelated issues, including incompressibility, the strong mean magnetic field limit, anisotropy of the variances (transverse fluctuations), anisotropy of the gradients (spectral anisotropy), and anisotropy of the system aspect ratio. Physical processes enter the considerations through their respective timescales, and these may depend on effects associated with turbulent Mach number, plasma beta $\beta_{\mathrm{p}}$, and wave propagation (sound speed $C_{s}$, Alfvén speed $V_{A}$ ). Ultimately this results in a separation of timescales and lengthscales, and these justify the mathematical steps that lead to RMHD.

\subsection{Primary Assumptions and Approximations}

The objective of the original derivations, due to KP and S76, was to find a simplified - but still nonlinearset of fluid equations relevant to the conditions present in tokamak plasmas. In particular, they both begin with low- $\beta_{\mathrm{p}}$ compressible $3 \mathrm{D} \mathrm{MHD}$ and the assumption of a strong mean magnetic field $B_{0} \hat{\boldsymbol{z}}$. Here, strong means that the energy in the mean field is much greater than that in the $\boldsymbol{v}$ and $\boldsymbol{b}$ fluctuations, i.e., the fluctuations are of small amplitude. A strong mean field is the fundamental assumption of RMHD.

The derivations proceed using perturbation theory based on postulated orderings of (i) the characteristic parallel and perpendicular lengthscales, and (ii) the fluctuation amplitudes: $\boldsymbol{v}_{\perp}, v_{\|}, \delta \rho$, etc. In KP the small parameter is the relative (transverse) fluctuation amplitude,

$$
\epsilon_{\mathrm{KP}}=b_{\perp} / B_{0} \ll 1,
$$

with $b_{\perp}$ the rms amplitude of $\boldsymbol{b}_{\perp}$. This is distinct from the small parameter employed in S76, namely the ratio of the perpendicular and parallel characteristic lengthscales,

$$
\epsilon_{\mathrm{S}}=\ell_{\perp} / \ell_{\|} \ll 1 \text {. }
$$

In order to obtain the RMHD model, it is not sufficient to simply require that one of these $\epsilon$ is smalladditional assumptions are needed. In particular, one needs to assume that the two ostensibly independent $\epsilon$ satisfy $\epsilon_{\mathrm{S}} \lesssim \epsilon_{\mathrm{KP}} \ll 1$, and are thus not fully independent. The necessity of two small parameters is not always made explicit in derivations.

Using slightly different sequences of arguments (see the Appendix), KP and S76 reason that the fluctuations of interest will have small components parallel to $\boldsymbol{B}_{0}$. Hence, the leading-order fluctuations are $\boldsymbol{v}_{\perp}$ and $\boldsymbol{b}_{\perp}$. Furthermore, these are transversely solenoidal, (e.g., $\nabla_{\perp} \cdot \boldsymbol{v}_{\perp}=0$ ), with their evolution governed by Eqns. (1)(2). Note that incompressibility was not imposed, but has emerged as a consequence of the assumptions made. Specific details of the S76 and KP derivations are summarized in the Appendix.

These approaches contain the essential description of RMHD. In each case, the rms transverse fluctuations, 
$\boldsymbol{v}_{\perp}$ and $\boldsymbol{b}_{\perp}$, are assumed similarly small in energy density (compared to the guide field energy $B_{0}^{2} / 2$ ). On its own, this small-amplitude assumption is often associated with the fluctuations being linear (shear) Alfvén waves. However, for RMHD, the aim is to support nonlinear effects at leading-order, and in fact the above orderings are consistent with turbulence, as becomes apparent in subsequent derivations (see below). In particular, $O(1)$ perpendicular lengthscales imply that the characteristic timescales of advective derivatives are of leading order.

\subsection{Timescale-based derivations}

Montgomery (M82) derived RMHD starting from an incompressible model. ${ }^{3}$ Rather than adopting a certain ordering of variables as a first step, his derivation examines a multiple-scales expansion of 3D MHD in terms of a small parameter $\epsilon$ that controls the strength of the mean magnetic field $B_{0}$. Formally this is achieved by replacing $\boldsymbol{B}_{0}$ with $\boldsymbol{B}_{0} / \epsilon$ in the full incompressible equations. ${ }^{4}$ Requirements are imposed on the expansion of the fluctuating fields such that the timescales of the solutionsincluding nonlinear effects and turbulence - remain $O(1)$ as $\epsilon \rightarrow 0$, i.e., as the strength of the mean field increases without bound. Thus, the expansion is developed in a way that eliminates fast timescales. This is the first derivation in which the enforcement of this condition was emphasized.

From the $O\left(\epsilon^{-1}\right)$ equations, one immediately finds that the parallel derivatives $\nabla_{z}$ of the leading-order fluctuations, $\boldsymbol{v}^{(0)}$ and $\boldsymbol{b}^{(0)}$, must be $O(\epsilon)$. This means that their variations with $z$ are "slow" compared to those in the $x$ and $y$ directions, and, correspondingly that in wavevector $(\boldsymbol{k})$ space there is spectral anisotropy: $k_{\|} \ll k_{\perp}$. It follows that to lowest order the transverse fluctuations are solenoidal in transverse planes: $\nabla_{\perp} \cdot \boldsymbol{v}_{\perp}^{(0)}=0$ and $\nabla_{\perp} \cdot \boldsymbol{b}_{\perp}^{(0)}=0$. Consequently, $\boldsymbol{v}_{\perp}^{(0)}$ and $\boldsymbol{b}_{\perp}^{(0)}$ can be expressed in terms of potentials $\phi$ and $A$ without loss of generality (as is also the case in KP and S76). Because of the spectral anisotropy, high-frequency Alfvén waves and high-frequency pseudo-Alfvén waves have been eliminated.

M82 is explicitly concerned with elimination of fast timescale motions, but assumes incompressibility from the outset. This leads to the same equations for $\boldsymbol{v}_{\perp}$ and $\boldsymbol{b}_{\perp}$ that KP and S76 obtain. However, because fast magnetosonic modes are never present in incompressible MHD, there is no need to assume the parallel fluctuations are weak compared to the perpendicular ones; that is, variance anisotropy is not a requirement in this derivation. Indeed, M82 finds that $v_{z}$ and $b_{z}$ enter at the same order as $\boldsymbol{v}_{\perp}$ and $\boldsymbol{b}_{\perp}$, whereas they enter at higher-order in $\mathrm{KP}$ and $\mathrm{S} 76$.

The KP and S76 derivations may also be interpreted in terms of an approximation in which the fast timescale dynamics have been eliminated. When $\beta_{\mathrm{p}} \ll 1$ (as they

3 Recall that in incompressible MHD, the two linearized wave modes of the system are the Alfvén and pseudo-Alfvén modes, respectively polarized in the toroidal $\boldsymbol{k} \times \boldsymbol{B}_{0}$ and poloidal $\boldsymbol{k} \times(\boldsymbol{k} \times$ $\left.\boldsymbol{B}_{0}\right)$ directions. Clearly, the Alfvén mode has no component in the parallel direction, whereas in general the pseudo-Alfvén mode does. Fast magnetosonic modes do not exist in incompressible MHD.

4 Montgomery's choice of $\epsilon$ is equivalent to KP's small parameter, $\epsilon_{\mathrm{KP}}$, since in Montgomery's case $b_{\perp}=O(1)$, and so $\epsilon=b_{\perp} / B_{0}$. both assume), two distinct types of anisotropy are needed to achieve this elimination. First, recall that fast magnetosonic modes are polarized such that $v_{z}$ and $b_{z}$ are nonzero. Since KP and S76 assume that $v_{z}$ and $b_{z}$ are one order of $\epsilon_{\mathrm{KP}}$ or $\epsilon_{\mathrm{S}}$ smaller than the perpendicular components, this means that fast magnetosonic modes are not present at the leading fluctuation order. Thus variance anisotropy is used to eliminate fast magnetosonic modes. (In fact, this eliminates fast modes of all frequencies.) Second, because Alfvén modes $\left(\omega= \pm k_{\|} V_{A}\right)$ and slow magnetosonic modes $\left(\omega \approx \pm k_{\|} C_{s}\right.$ when $\left.\beta_{\mathrm{p}}<1\right)$ have anisotropic dispersion relations, lengthscale anisotropy $\left(\epsilon_{\mathrm{S}}=\ell_{\perp} / \ell_{\|} \ll 1\right)$ or equivalently spectral anisotropy $\left(k_{\|} \ll k_{\perp}\right)$ ensures these modes are of low-frequency, if present.

Another derivation that emphasizes the removal of fast timescales, this time beginning with the compressible equations, is due to ZM92. They employ what is ostensibly a further small parameter, the Alfvén Mach number, $M_{A}=u_{0} / V_{A}\left(u_{0}\right.$ is a characteristic fluctuation speed). However, for RMHD $u_{0} \approx b_{\perp}$, in Alfvén speed units, and thus $M_{A} \approx b_{\perp} / B_{0}=\epsilon_{\mathrm{KP}}$ is not a distinct expansion parameter.

Recall that the physical content of an RMHD derivation based on timescales is that the characteristic nonlinear timescale, $\tau_{\mathrm{NL}}$, should be no slower than the wave timescales, e.g., for Alfvén waves with timescale $\tau_{\mathrm{A}}$,

$$
\tau_{\mathrm{NL}} \lesssim \tau_{\mathrm{A}}
$$

When $\beta_{\mathrm{p}}$ is $O(1)$ or smaller, this can be achieved by imposing restrictions on the characteristic lengthscales along and across $\boldsymbol{B}_{0}$ (i.e., spectral anisotropy). Using standard definitions in Eq. (5), we have $\ell_{\perp} / \delta b \lesssim \ell_{\|} / B_{0}$, and with $\delta b \approx b_{\perp}$ this implies $\epsilon_{\mathrm{S}} \lesssim \epsilon_{\mathrm{KP}}$. Thus, two essential assumptions for obtaining RMHD from compressible 3D MHD can be combined and written as

$$
\frac{\ell_{\perp}}{\ell_{\|}} \lesssim \frac{b_{\perp}}{B_{0}} \ll 1 \quad \text { or } \quad \epsilon_{\mathrm{S}} \lesssim \epsilon_{\mathrm{KP}} \ll 1 .
$$

For the $\beta_{\mathrm{p}} \leq 1$ case, elimination of the high-frequency slow modes and the high-frequency Alfvén modes imposes no further restriction. The complete elimination of high-frequency fast modes $\left(\omega \approx k \sqrt{V_{A}^{2}+C_{s}^{2}}\right)$, however, requires that the fluctuations are strictly transverse, i.e., $v_{z}=0=b_{z}$ (ZM92).

The ZM92 derivation is related to the development of so-called Nearly Incompressible MHD (Zank \& Matthaeus 1993), but differs in that it is not based on a perturbation series but instead employs Kreiss's principle to eliminate fast timescales by bounding time derivatives in the initial data (Kreiss 1980). We refer the reader to the original paper for details of this development, but the important conclusion is that for both $\beta_{\mathrm{p}}=O(1)$ and for $\beta_{\mathrm{p}} \ll 1$ the RMHD equations are as given above in Eqs. (1) and (2), with no parallel fluctuations present.

\subsection{System size and characteristic lengths}

The relationship between the size of a physical system and the lengthscales characteristic of RMHD dynamics is not always straightforward. For the case of a finite size domain the lengthscales $\ell_{\|}$and $\ell_{\perp}$ may sometimes be associated with the dimensions of the container $\left(L_{\perp}\right.$ 
and $L_{\|}$, say), as in Strauss's (S76) rectangular idealization of a tokamak. The lengthscale ratio is then also the aspect ratio of the device. However this identification may not always be dynamically meaningful. For idealized tokamaks with very strong guide field, or similar cases relating to solar flux tubes with certain boundary conditions, it may be necessary to distinguish between the system $\left(L_{\|}\right)$and characteristic $\left(\ell_{\|}\right)$lengths. ${ }^{5}$ This may occur when the mean magnetic field is so strong that $b_{\perp} / B_{0}$ is smaller than the inverse aspect ratio. It is then possible that the "non-propagating mode" with zero $\nabla_{\|}$(and arbitrary $\perp$ structure) is the only surviving class of fluctuations that obeys the conditions in Eqs. (5) and (6). These fluctuations, with $\tau_{\mathrm{A}} \rightarrow \infty$ and $\ell_{\|} \rightarrow \infty$, always comply with these conditions. In this way one sees that the $2 \mathrm{D}$ modes are the core RMHD fluctuations, as discussed in ZM92; see also Dmitruk et al. (1998).

\subsection{Role of $\beta_{p}$}

It is evident that the size of $\beta_{\mathrm{p}}$ impacts on the RMHD approximation. The key point is that, at leading-order, Eqs. (1)-(2) for the transverse fluctuations are valid for all $\beta_{\mathrm{p}}$, whereas most derivations find that parallel fluctuations are absent at $\beta_{\mathrm{p}} \leq 1$, but may be present for large $\beta_{\mathrm{p}}$. Below we provide some expanded discussion on $\mathrm{RMHD}$ in these different $\beta_{\mathrm{p}}$ regimes.

KP's derivation ignores mechanical pressure and thus assumes $\beta_{\mathrm{p}} \rightarrow 0$. While this simplifies the physical picture in some ways, it complicates the approach to incompressibility, since only magnetic pressure is present to enforce the condition $\frac{\partial}{\partial t}(\nabla \cdot v)=0$. In S76, the mechanical pressure is retained, at $O\left(\epsilon_{\mathrm{S}}^{2}\right)$, and since $B_{0}=O(1)$, one has $\beta_{\mathrm{p}}=O\left(\epsilon_{\mathrm{S}}^{2}\right)$. Both of these approaches are low $\beta_{\mathrm{p}}$, and the fluctuations have strictly transverse polarizations. ${ }^{6}$

In the M82 derivation, incompressibility is assumed from the outset and $\beta_{\mathrm{p}}$ is not discussed. Presumably this (incompressible) model applies to plasma with low Mach number and high $\beta_{\mathrm{p}}$. However, under these conditions the approach to incompressibility does not require a collapse of dimensionality, and one can recover an intrinsically isotropic MHD fluid model (Zank \& Matthaeus 1993). A strong magnetic field is still imposed, but not strong enough to violate the assumption of high $\beta_{\mathrm{p}}$. That is we must maintain $V_{A} \ll C_{s}$. In this case, the strong magnetic field expansion does not impose variance anisotropy, and the presence of parallel variances$v_{z}$ and $b_{z}$ - does not violate the assumption that dynamical speed remain slow compared to the acoustic (or fast magnetosonic) wave speeds. Nevertheless the mismatch between advective or turbulence timescales and much faster high- $k_{\|}$Alfvén speeds does induce a wavevector anisotropy, as described by Shebalin et al. (1983) [see also Bondeson (1985); Grappin (1986); Oughton et al. (1994)].

It follows that an RMHD limit can be achieved at high $\beta_{\mathrm{p}}$, but it necessarily involves two distinct stages: The first involves a reduction to the incompressible limit, usually attained by imposing a low Mach number ordering,

\footnotetext{
5 This is certainly the case for homogeneous turbulence.

6 See Strauss (1977) for a demonstration that in the $\beta_{\mathrm{p}}=O\left(\epsilon_{\mathrm{S}}\right)$ case parallel variances can be taken equal to zero.
}

together with bounded compressible fluctuations in initial data or forcing (see e.g., Klainerman \& Majda 1982; Matthaeus \& Brown 1988). Second, the strong but intermediate valued mean magnetic field $\left(v_{\perp}, b_{\perp} \ll V_{A} \ll\right.$ $C_{s}$ ) mandates that one also requires $k_{\|} \ll k_{\perp}$ spectral anisotropy, in order to restrict time variations to be at advective or turbulence timescales, such as $\ell_{\perp} / v_{\perp}$.

Schekochihin et al. (2009) found that parallel variances (and density fluctuations) are part of RMHD for all values of $\beta_{\mathrm{p}}$. One reason for this is their assumption that all fluctuations enter at order $\epsilon=k_{\|} / k_{\perp} \ll 1$, whereas in KP and S76 the parallel fluctuations are an order smaller (as are the density and pressure fluctuations). Moreover, although fast mode timescales are set aside in their development, it is not clear that their absence can be maintained since the fast mode polarization involves parallel variances and these are allowed under the Schekochihin et al. (2009) ordering. They suggest strong collisionless damping of fast modes and weak transfer of their energy to high-frequency Alfvén modes as factors supporting the irrelevance of such modes. However, since these processes depend on $\beta_{\mathrm{p}}$ it is unclear whether it is consistent to retain parallel fluctuations (at leading order) for all $\beta_{\mathrm{p}}$.

Summarizing, the major role that $\beta_{\mathrm{p}}$ plays in RMHD is determining whether or not parallel variances are present (at leading order). The transverse components of the fluctuations obey Eqs. (1)-(2) for all values of $\beta_{\mathrm{p}}$. For systems with $\beta_{\mathrm{p}} \ll 1$ or $\beta_{\mathrm{p}} \approx 1$, the RMHD approximation does not have parallel fluctuations (KP, S76, ZM92). However, when the initial system is incompressible, or when $\beta_{\mathrm{p}} \gg 1$, the RMHD approximation can include $v_{z}$ and $b_{z}$ fluctuations at the same order as the $\boldsymbol{v}_{\perp}$ and $\boldsymbol{b}_{\perp}$ ones. These parallel fluctuations obey "passive" linear equations (M82; Kinney \& McWilliams 1998). See Kinney \& McWilliams (1997); Kruger et al. (1998) for analogous discussions of the role and dynamics of parallel variances in other formulations that are related to RMHD.

Note that in contrast to this $\beta_{\mathrm{p}}$-dependent aspect of variance anisotropy, spectral anisotropy is an inherent requirement for RMHD - whatever the value of $\beta_{\mathrm{p}}$ - since otherwise high-frequency Alfvénic motions could arise.

\section{STRUCTURE OF THE RMHD EQUATIONS}

The above review of derivations highlights that there are two small parameters of importance in RMHD- $\epsilon_{\mathrm{KP}}$ and $\epsilon_{\mathrm{S}}$ - and suggests that a comprehensive derivation of the RMHD equations can be achieved using a multiplescales analysis that is based on both of these. ${ }^{7}$ Thus, let us define a slowly-varying parallel coordinate $s^{\prime}=\epsilon_{\mathrm{S}} z$ and a fast time coordinate $\tau^{\prime}=t / \epsilon_{\mathrm{KP}}$, and treat all fluctuations as functions of these and the original coordinates, e.g., $\boldsymbol{b}\left(x, y, z, s^{\prime}, t, \tau^{\prime}\right)$. The fields themselves are assumed of the form:

$$
\begin{aligned}
\boldsymbol{B}_{0}+\boldsymbol{b} & =\frac{B_{0}^{\prime} \hat{z}}{\epsilon_{\mathrm{KP}}}+\boldsymbol{b}^{\prime}+\epsilon_{\mathrm{KP}} \boldsymbol{b}_{1}^{\prime}+\ldots \\
\boldsymbol{v} & =\boldsymbol{v}^{\prime}+\epsilon_{\mathrm{KP}} \boldsymbol{v}_{1}^{\prime}+\ldots
\end{aligned}
$$

A prime superscript $\left({ }^{\prime}\right)$ denotes rescaled quantities (with magnitudes of order unity), sometimes referred to

\footnotetext{
7 Multiple-scales approaches were employed in M82 and Kruger et al. (1998), but in each case based on only one of $\epsilon_{\mathrm{KP}}$ or $\epsilon_{\mathrm{S}}$.
} 
as being in code units for RMHD. The unprimed variables on the LHSs are in physical or laboratory units. Density and pressure are expanded in similar ways, although it is necessary to consider $\beta_{\mathrm{p}}$ in their expansions (Zank \& Matthaeus 1992, 1993). The $x, y$, and $t$ coordinates are left unscaled. This $O(1)$ scaling of the 'main' coordinates, along with the leading-order fluctuations also being $O(1)$, is a convenient property of the chosen ordering scheme, particularly for turbulence applications.

Substituting into the compressible 3D MHD equations one then demands that time derivatives are $O(1)$ - i.e., that $\partial / \partial \tau^{\prime}=0$ - so that all high-frequency fluctuations are eliminated. ${ }^{8}$ One finds that at leading-order, $\boldsymbol{v}^{\prime}$ and $\boldsymbol{b}^{\prime}$ are transverse to $\boldsymbol{B}_{0}^{\prime}$ and also solenoidal. Thus, $\nabla_{\perp} \cdot \boldsymbol{v}^{\prime}=0$ and $\boldsymbol{v}^{\prime} \cdot \boldsymbol{B}_{0}^{\prime}=0$, for example, where $\nabla_{\perp}=\hat{\boldsymbol{x}} \partial_{x}+\hat{\boldsymbol{y}} \partial_{y}$. Moreover, although the fluctuations depend upon all three spatial coordinates, the leadingorder dependence on the parallel coordinate is intrinsically weak (aka slowly-varying); that is, $\boldsymbol{v}^{\prime}$ and $\boldsymbol{b}^{\prime}$ are functions of $s^{\prime}$ but not of $z$. Thus RMHD has a restricted $3 \mathrm{D}$ dependence, rather than a "fully 3D" dependence (see $\S 5)$.

It is helpful to express the RMHD equations in terms of transverse ${ }^{9}$ Elsasser variables, $\boldsymbol{z}^{ \pm}\left(x, y, s^{\prime}, t\right)=\boldsymbol{v}^{\prime} \pm$ $\boldsymbol{b}^{\prime} / \sqrt{4 \pi \rho}$, where the mass density $\rho$ is taken to be uniform. One obtains

$$
\begin{aligned}
\frac{\partial \boldsymbol{z}^{ \pm}}{\partial t} \mp\left(\frac{V_{A}^{\prime}}{\epsilon_{\mathrm{KP}}}\right)\left(\epsilon_{\mathrm{S}} \frac{\partial}{\partial s^{\prime}}\right) \boldsymbol{z}^{ \pm}= & -\boldsymbol{z}^{\mp} \cdot \nabla_{\perp} \boldsymbol{z}^{ \pm} \\
& -\nabla_{\perp} p^{*}+\nu \nabla_{\perp}^{2} \boldsymbol{z}^{ \pm} \\
& +O\left(\epsilon_{\mathrm{KP}}\right)+O\left(\epsilon_{\mathrm{S}}\right),
\end{aligned}
$$

with $V_{A}^{\prime}=B_{0}^{\prime} / \sqrt{4 \pi \rho}$ and $p^{*}$ the mechanical plus magnetic pressure. ${ }^{10}$ For simplicity, viscosity $\nu$ and resistivity $\eta$ are assumed equal.

A key feature of RMHD - the lack of high-frequency Alfvén waves - is associated with the second term on the LHS of Eq. (9). Physically, the bracketed pieces in this 'wave term' correspond to the actual (very large) mean Alfvén speed and the actual (very weak) gradient operator in the parallel direction. The RMHD approximation is such that these offset each other to produce an at most $O(1)$ variation. Hence there are no high-frequency Alfvén waves in RMHD. Low-frequency Alfvén waves typically are present.

The 'wave term' is usually written as $V_{A}^{\prime \prime} \partial \boldsymbol{z}^{ \pm} / \partial s^{\prime}$, where $^{11}$

$$
V_{A}^{\prime \prime}=V_{A}^{\prime} \frac{\epsilon_{\mathrm{S}}}{\epsilon_{\mathrm{KP}}}=V_{A}^{\prime} \frac{\tau_{\mathrm{NL}}}{\tau_{\mathrm{A}}}
$$

is the mean Alfvén speed in rescaled (code) units - not physical units (see §3.5). In writing it with the $\epsilon_{\mathrm{S}} / \epsilon_{\mathrm{KP}}$

\footnotetext{
8 Operationally, one can take earlier derivations such as KP, S76, or ZM92, and replace all occurrences of $\partial / \partial z$ with $\left(\epsilon_{\mathrm{S}} / \epsilon_{\mathrm{KP}}\right) \partial / \partial s^{\prime}$.

9 As discussed in $\S 2.5$, if $\beta_{\mathrm{p}} \gg 1$ then parallel components of the fluctuations may also be present at the same order (M82; Kinney \& McWilliams 1997; Schekochihin et al. 2009). However, they obey linear equations and are of less interest here.

10 For numerical solutions, other forms of the RMHD equations are more efficient. These typically solve for the (parallel) components of the vector potential and vorticity. See Eqs. (19)-(20).

11 The double primes on $V_{A}^{\prime \prime}$ serve as a reminder that (a) both small parameters enter into its definition, and (b) it is a rescaled quantity.
}

ratio explicit, we are emphasizing how the two small parameters interact (M82; Oughton et al. 2004). Interestingly, $\epsilon_{\mathrm{KP}} / \epsilon_{\mathrm{S}}=\left(b_{\perp} / B_{0}\right)\left(\ell_{\|} / \ell_{\perp}\right)$ is formally the same as the Kubo (1963) number, $K$; see $\S 3.4$.

As is well-known, the gradient operator in Eq. (9) is the transverse one, acting only on the $x$ and $y$ coordinates. Notably, there is no parallel dissipation in RMHD (M82). This is an important difference from 3D MHD and its ramifications are considered in $\S 4.1$. At leadingorder, parallel gradients, $\partial / \partial s^{\prime}$, appear only in the wave operator.

As is usual for incompressible flows, the pressure is determined from a Poisson equation, obtained from the (transverse) divergence of Eq. (9):

$$
\nabla_{\perp}^{2} p_{\perp}^{*}=-\nabla_{\perp} \cdot\left(\boldsymbol{z}^{\mp} \cdot \nabla_{\perp} \boldsymbol{z}^{ \pm}\right) .
$$

The pressure gradient force can be viewed as a projection operator which enforces incompressibility. In doing so, the pressure couples the $x$ and $y$ components of $\boldsymbol{z}^{ \pm}$, thereby enabling transfer of energy between them. Indeed, in the simpler case of incompressible Navier-Stokes turbulence, the pressure acts to isotropize the energy in the cartesian components of the velocity (Batchelor 1970). The extent to which this tendency towards variance isotropy occurs in (R)MHD is an interesting question.

\subsection{Fourier Space}

When investigating the linear and nonlinear features of RMHD turbulence it can be helpful to work in Fourier space. If $\boldsymbol{k}=\boldsymbol{k}_{\perp}+\boldsymbol{\kappa}_{\|}^{\prime} \hat{\boldsymbol{B}}_{0}$ is the Fourier wavevector, then the Fourier transform of Eq. (9) is

$$
\begin{aligned}
\left(\frac{\partial}{\partial t} \mp i \kappa_{\|}^{\prime} V_{A}^{\prime \prime}\right) \boldsymbol{z}^{ \pm}(\boldsymbol{k})= & -i \int \boldsymbol{z}^{\mp}(\boldsymbol{p}) \cdot \boldsymbol{q}_{\perp} \boldsymbol{z}^{ \pm}(\boldsymbol{q}) \mathrm{d} \boldsymbol{p} \mathrm{d} \boldsymbol{q} \\
& -i \boldsymbol{k}_{\perp} p^{*}(\boldsymbol{k})-\nu k_{\perp}^{2} \boldsymbol{z}^{ \pm}(\boldsymbol{k}), \quad(12)
\end{aligned}
$$

where the integration is subject to the wavevector triad constraint $\boldsymbol{k}=\boldsymbol{p}+\boldsymbol{q}$. Note that the parallel component of $\boldsymbol{k}$ is written $\kappa_{\|}^{\prime}\left(\right.$ not $\left.k_{\|}\right)$as a reminder that this is a slowly-varying coordinate, Fourier conjugate to $s^{\prime}$. The symbol $k_{\|}$is reserved for the 3D MHD situation where no rescaling of coordinates is performed.

\subsection{Slab Modes}

Fourier modes with $\boldsymbol{k} \| \boldsymbol{B}_{0}$ (i.e, $k_{\perp}=0$ ) are often referred to as slab modes, since they have no transverse structuring. From Eq. (12) one can show that these obey linear equations. This follows because the first term on the RHS can be rewritten as proportional to $\boldsymbol{k}_{\perp}$, using $\boldsymbol{q}_{\perp}=\boldsymbol{k}_{\perp}-\boldsymbol{p}_{\perp}$. So when $k_{\perp}=0$, all terms on the RHS vanish leaving only linear (and undamped!) evolution of the slab modes. The linearity also means that such modes cannot be dynamically generated in RMHD.

The question remains, however, of whether slab modes are allowable in RMHD initial conditions. Formally, each slab mode has a (modal) nonlinear time which is infinite, and thus the RMHD timescale restriction, Eq. (5), cannot be satisfied for slab modes. On the other hand, one might consider comparing a slab mode's Alfvén time, $\tau_{\mathrm{A}}\left(\kappa^{\prime}\right)$ to the nonlinear time associated with all modes on the $k$-space shell $|\boldsymbol{k}|=\kappa^{\prime}: \tau_{\mathrm{NL}}(k) \approx 1 /\left(k u_{k}\right)$, where $u_{k}$ is 
the rms speed for fluctuations with scales $\sim 1 / k$. But in this case, $\tau_{\mathrm{NL}}\left(\kappa^{\prime}\right) \lesssim \tau_{\mathrm{A}}\left(\kappa^{\prime}\right)$ is equivalent to $B_{0} \lesssim u_{\kappa^{\prime}}$ which violates the fundamental strong mean field assumption of RMHD. Thus, slab modes are absent in RMHD.

\subsection{Connection with toroidal and poloidal polarizations}

RMHD's transverse incompressibility, $\boldsymbol{k}_{\perp} \cdot \boldsymbol{z}^{ \pm}(\boldsymbol{k})=0$, means that the fluctuations are polarized perpendicular to $\boldsymbol{k}_{\perp}$. Moreover, since the (leading-order) fluctuations have no parallel components, $\boldsymbol{z}^{ \pm}(\boldsymbol{k})$ must be parallel to $\boldsymbol{k}_{\perp} \times \hat{\boldsymbol{B}}_{0}$, which is the toroidal direction. Thus RMHD fluctuations are toroidally polarized. This is perhaps most easily seen in Fourier space, as shown below.

Recall that for each wavevector $\boldsymbol{k}$, there are two linearly independent basis vectors that span the plane with $\boldsymbol{k}$ as a normal vector. A physically motivated choice of unit basis vectors is the toroidal and poloidal decomposition, $\boldsymbol{e}_{T}(\boldsymbol{k})=\boldsymbol{k} \times \hat{\boldsymbol{B}}_{0} / k_{\perp}$ and $\boldsymbol{e}_{P}(\boldsymbol{k})=\hat{\boldsymbol{k}} \times \boldsymbol{e}_{T} \cdot{ }^{12}$ As is well-known, these unit vectors coincide with the polarization directions for the linearized wave modes of incompressible 3D MHD: $\boldsymbol{e}_{T}$ for the shear Alfvén wave and $\boldsymbol{e}_{P}$ for the pseudo-Alfvén wave. The incompressible MHD equations are readily analyzed in terms of this basis as has been frequently discussed (e.g., Craya (1958); Herring (1974); Krause \& Rädler (1980); Schmidtmann et al. (1998); Howes (2015)).

Taking the dot product of Eq. (12) with $\boldsymbol{e}_{T}(\boldsymbol{k})$ and writing $z_{\text {rmhd }}^{ \pm}(\boldsymbol{k})=\boldsymbol{e}_{T}(\boldsymbol{k}) \cdot \boldsymbol{z}^{ \pm}(\boldsymbol{k})$ yields

$$
\begin{aligned}
\left(\frac{\partial}{\partial t} \mp i \kappa_{\|}^{\prime} V_{A}^{\prime \prime}\right) & z_{\mathrm{rmhd}}^{ \pm}(\boldsymbol{k})=-\nu k_{\perp}^{2} z_{\mathrm{rmhd}}^{ \pm}(\boldsymbol{k}) \\
& -i \int \boldsymbol{z}^{\mp}(\boldsymbol{p}) \cdot \boldsymbol{q}_{\perp} \boldsymbol{z}^{ \pm}(\boldsymbol{q}) \cdot \boldsymbol{e}_{T}(\boldsymbol{k}) \mathrm{d} \boldsymbol{p} \mathrm{d} \boldsymbol{q} .
\end{aligned}
$$

The pressure gradient term has dropped out since it acts parallel to $\boldsymbol{k}_{\perp}$, and $\boldsymbol{k}_{\perp} \cdot \boldsymbol{e}_{T}(\boldsymbol{k})=0$.

Of course, incompressible 3D MHD can also be written in terms of poloidal and toroidal components, and it is of interest to understand its relationship to RMHD. As the above discussion suggests, the primary distinction hinges on the presence or absence of poloidal fluctuations, although the dissipation terms also differ. Working with the full 3D Elsasser variables $\boldsymbol{z}_{3 \mathrm{D}}^{ \pm}(\boldsymbol{k}, t)$ - so that the parallel coordinate is not restricted to large-scale variations - and writing now $\boldsymbol{k}=\left(k_{x}, k_{y}, k_{\|}\right)$,

$$
\begin{aligned}
\boldsymbol{z}_{3 \mathrm{D}}^{ \pm}(\boldsymbol{k}) & =\boldsymbol{e}_{T}(\boldsymbol{k}) z_{T}^{ \pm}(\boldsymbol{k})+\boldsymbol{e}_{P}(\boldsymbol{k}) z_{P}^{ \pm}(\boldsymbol{k}) \\
& =\boldsymbol{z}_{T}^{ \pm}(\boldsymbol{k})+\boldsymbol{z}_{P}^{ \pm}(\boldsymbol{k}),
\end{aligned}
$$

one can employ this form with the equations of incompressible 3D MHD given a mean magnetic field with associated Alfvén speed $V_{A}^{3 \mathrm{D}}$. Note that no rescaling of coordinates or fields occurs. Setting all $z_{P}^{ \pm}(\boldsymbol{k})=0$ then yields

$$
\begin{aligned}
\left(\frac{\partial}{\partial t} \mp i k_{\|} V_{A}^{3 \mathrm{D}}\right) & z_{T}^{ \pm}(\boldsymbol{k})=-\nu k^{2} z_{T}^{ \pm}(\boldsymbol{k}) \\
& -i \int \boldsymbol{z}_{T}^{\mp}(\boldsymbol{p}) \cdot \boldsymbol{q}_{\perp} \boldsymbol{z}_{T}^{ \pm}(\boldsymbol{q}) \cdot \boldsymbol{e}_{T}(\boldsymbol{k}) \mathrm{d} \boldsymbol{p} \mathrm{d} \boldsymbol{q},
\end{aligned}
$$

${ }^{12}$ When $\boldsymbol{k} \| \boldsymbol{B}_{0}$ one can take $\boldsymbol{e}_{T}=\hat{\boldsymbol{x}}$ and $\boldsymbol{e}_{P}=\hat{\boldsymbol{y}}$. where the integration is again subject to the triad constraints $\boldsymbol{k}=\boldsymbol{p}+\boldsymbol{q}$.

Comparing Eqs. (13) and (16) it is clear that the incompressible 3D MHD equations with all poloidal fluctuations set to zero are formally equivalent to the RMHD equations - except for their retention of parallel dissipation, i.e., the dissipation terms are proportional to $k^{2}$ (versus the $k_{\perp}^{2}$ of RMHD). The absence of parallel dissipation in RMḦD has important consequences for spectral transfer in the parallel direction; see $\$ 4.1$.

However, this formal (near) equivalence masks the inherent anisotropy of RMHD. In RMHD $s^{\prime}$ (and $\kappa_{\|}^{\prime}$ ) are large-scale coordinates, compared to the transverse ones, and $V_{A}^{\prime \prime}$ is a rescaled quantity. No such restrictions are made in obtaining Eq. (16). In particular, $1 / k_{\|}$is not required to be a large scale, and $V_{A}^{3 \mathrm{D}}$ is of arbitrary strength (i.e., need not be strong). Indeed it is well known from numerical experiments (Dmitruk et al. 2005; Oughton et al. 2016) that, for $\delta b / B_{0} \sim 1$, dynamical evolution beginning with a "toroidal-only" (or transverse) initial condition, will lead to significant $O(1)$ parallel (poloidal) fluctuations in just an eddy turnover time. Therefore, in general terms, the toroidal-only formulation is grossly unstable unless the RMHD requirements are met.

\subsection{Kubo number}

In his investigation of stochastic Liouville equations, Kubo (1963) introduced a dimensionless parameter measuring the strength of perturbations in such systems, subsequently referred to as the Kubo number, $K$. For field-line transport in frozen magnetic turbulence it can be written as $K=\left(\delta b / B_{0}\right)\left(\ell_{\|} / \ell_{\perp}\right)$ where $B_{0}$ is the average field, $\delta b$ is the rms fluctuating field, and $\ell_{\|}\left(\ell_{\perp}\right)$ is the correlation length parallel (perpendicular) to $\boldsymbol{B}_{0}$. For RMHD, field-line transport depends only upon $K$ (Snodin et al. 2013; Servidio et al. 2014). In the RMHD context (where $\delta b=b_{\perp}$ ) one also has $K=\epsilon_{\mathrm{KP}} / \epsilon_{\mathrm{S}}$, which is the ratio of two small parameters and so its size may not be immediately apparent. ${ }^{13}$

When the RMHD approximation is valid, $\epsilon_{\mathrm{KP}}$ and $\epsilon_{\mathrm{S}}$ must both be small, and they must satisfy Eq. (6). Rearranging the latter one obtains

$$
1 \lesssim K \ll \frac{\ell_{\|}}{\ell_{\perp}} .
$$

Thus, in a system obeying the RMHD equations, the Kubo number is constrained to lie between roughly unity and about a tenth of $\ell_{\|} / \ell_{\perp}$. The latter condition, however, is automatically satisfied if the approximations leading to RMHD are also satisfied, (i.e., $\epsilon_{\mathrm{KP}}, \epsilon_{\mathrm{S}} \ll 1$ ), so that the relevant restriction is $K \gtrsim 1$.

The only place the Kubo number appears in the RMHD equations is in the 'wave term'; see Eq. (9). However, this appearance is usually hidden via use of Eq. (10):

$$
\frac{V_{A}^{\prime}}{K} \frac{\partial \boldsymbol{z}^{ \pm}}{\partial s^{\prime}}=V_{A}^{\prime \prime} \frac{\partial \boldsymbol{z}^{ \pm}}{\partial s^{\prime}} .
$$

Here, $V_{A}^{\prime \prime}$ is the Alfvén speed associated with the mean

13 In non-RMHD contexts, $K$ might not involve small parameters. 
magnetic field, in code units (see next section).

\subsection{Units, numerics, and rescaling of $V_{A}^{\prime \prime} \frac{\partial}{\partial s^{\prime}}$}

The distinction between physical units and code units can induce some confusion when using the RMHD equations, including determining correct physical values for $\epsilon_{\mathrm{KP}}=b_{\perp} / B_{0}$. A point to keep firmly in mind is that, physically, $\epsilon_{\mathrm{KP}}$ must be small, since this is a necessary condition for validity of the RMHD approximation.

Suppose that one wishes to solve the RMHD equations numerically. A first step is to decide whether to employ the equations written in physical ${ }^{14}$ units or those written in rescaled 'code' units. Denoting the rms amplitudes of the (transverse) fluctuations by $\delta b$ and $\delta v$, the former case has $\delta b, \delta v \ll B_{0}$ and $\ell_{\perp} \ll \ell_{\|}$. In the case of code units, it is often convenient to choose both $\delta b, \delta v \approx 1$ and $V_{A}^{\prime \prime} \approx 1$. Naively, the later choice would appear to involve $\delta b / V_{A}^{\prime \prime} \approx 1$ (where $V_{A}^{\prime \prime} \equiv B_{0}^{\prime \prime}$ ), but this is an incorrect interpretation since $V_{A}^{\prime \prime}$ is a rescaled quantity [see Eqs. (9), (10), and (18)].

Examination of the wave term in the RMHD equations, Eqs. (9)-(10), reveals that it has a rescaling symmetry: allowing $V_{A}^{\prime \prime} \rightarrow \alpha V_{A}^{\prime \prime}$ and $\frac{\partial}{\partial s^{\prime}} \rightarrow \frac{1}{\alpha} \frac{\partial}{\partial s^{\prime}}$ leaves the wave term invariant. In other words, increasing the mean field strength by a factor $\alpha$ while simultaneously scaling the parallel coordinate by the same factor leaves the equations unchanged (e.g., Beresnyak 2012)

Computationally, this means that a single simulation corresponds to (infinitely) many physical systems, each with a different strong $V_{A}$. For example, a numerical setup might employ $V_{A}^{\prime \prime}=1$ and $N_{z}$ (uniformly spaced) gridpoints in the $\boldsymbol{B}_{0}$ direction. At any time during the simulation, the parallel correlation length may be calculated. Suppose it is determined to be $\ell_{\|}^{\text {cor }}=\Lambda_{\|}$in nondimensional code units. This can be interpreted as corresponding to a physical system with $V_{A}=\alpha$ and $\ell_{\|}^{\text {cor }}=\alpha \Lambda_{\|}$, for each $\alpha \gg 1$ (where $\alpha$ must be large so that the assumptions on which RMHD is based are satisfied). Rescalings of this kind do not alter the Kubo number.

The form of the dissipation operators is also influenced by the choice of physical units or numerical units. In code units, standard scalar dissipation (viscous and resistive) involves the perpendicular Laplacian, $\nabla_{\perp}^{2}$, not the 3D Laplacian. Use of the later would correspond to a very large parallel viscosity. See the final paragraph of $\S 4.1$.

Finally in this section we note that when solving the RMHD equations numerically, it is often advantageous to express them as evolution equations for the parallel components of the magnetic vector potential, $a_{z} \equiv a$, and the fluid vorticity $\omega=\hat{\boldsymbol{z}} \cdot \nabla_{\perp} \times \boldsymbol{v}$ (e.g., S76, M82). In our notation these equations are

$$
\begin{aligned}
& \frac{\partial a}{\partial t}+\boldsymbol{v} \cdot \nabla_{\perp} a=V_{A}^{\prime \prime} \frac{\partial \phi}{\partial s^{\prime}}+\eta \nabla_{\perp}^{2} a, \\
& \frac{\partial \omega}{\partial t}+\boldsymbol{v} \cdot \nabla_{\perp} \omega=V_{A}^{\prime \prime} \frac{\partial j}{\partial s^{\prime}}+\boldsymbol{b} \cdot \nabla_{\perp} j+\nu \nabla_{\perp}^{2} \omega,
\end{aligned}
$$

where the electric current density $j=-\nabla_{\perp}^{2} a$ and $\phi$ is the stream function satisfying $\omega=-\nabla_{\perp}^{2} \phi$. These are

14 Here, physical means a standard dimensionless form of the equations, but with no small parameters identified. of course equivalent to the 'primitive' variable forms, Eqs. (1)-(2). They do, however, afford considerable gains in numerical efficiency since there are only two equations to solve as opposed to the four equations of the primitive variable case. In addition, there is no need to solve for the pressure.

\subsection{Boundary Conditions}

Often in RMHD applications (e.g., coronal loops), the system is not periodic in the $\boldsymbol{B}_{0}$ direction. Consequently, the boundary conditions (BCs) on the outer surfaces of the system (in the $\boldsymbol{B}_{0}$ direction), say, $s^{\prime}=0$ and $s^{\prime}=L$, need to be of a more general type. The possibility that these BCs induce boundary layers adjacent to the end surfaces then needs to be considered (Scheper \& Hassam 1999). In particular, one would like to know whether the RMHD model remains valid in these putative boundary layers, or whether they are associated with structure in $s^{\prime}$ that is of such small scale that the RMHD lengthscale inequality $\ell_{\perp} / \ell_{\|} \ll 1$ is violated [see Eq. (4)].

A class of BCs relevant to coronal applications is the line-tied ones, meaning that the normal component of the magnetic field is continuous across the boundary(s), and that it moves with the transverse flow at the boundary. When line-tied BCs are employed, and also the system is quasistatic, Scheper \& Hassam (1999) report that boundary layers do form, but that the transverse $\boldsymbol{v}$ and $\boldsymbol{b}$ are essentially unaffected with their $s^{\prime}$ dependence still satisfying the RMHD assumptions. There is, however, a correction to the parallel magnetic field which has $\partial_{z} \sim 1 / \ell_{\perp}$ and this needs to be taken into account.

Line-tied BCs are also of interest in turbulence (i.e., non-quasistatic) RMHD studies, where the velocity fluctuations obey a dynamical equation. Results in Rappazzo et al. (2008) suggest that the RMHD model remains valid in these situations, with all quantities being slowly-varying with respect to the parallel coordinate $z$. We are not aware of any reports on the development of boundary layers given these BCs.

Other types of BC were investigated by Dmitruk et al. (2001), where Dirichlet and Neumann conditions were imposed on the velocity stream function and magnetic potential. ${ }^{15}$ Here too, there was no indication of boundary layers forming. Thus we conclude, that at least for physically consistent $\mathrm{BCs}$, the RMHD model remains valid for many different types of BCs.

\section{DYNAMICAL ISSUES}

\subsection{Parallel spectral transfer and dissipation}

Energy does spread in the $k_{\|}$direction in RMHD (Kinney \& McWilliams 1998; Oughton et al. 2004; Dmitruk et al. 2005). However because of the inherent lengthscale anisotropy of RMHD, and because of the lack of parallel variance of the fluctuations, this parallel spectral transfer is weak. There are in fact no 'parallel nonlinearity' terms like $b_{\|} \nabla_{\|} \boldsymbol{b}$ in the equations; the absence of poloidal (i.e., non-Alfvénic) fluctuations ensures that such terms cannot appear. So, even though there are triadic couplings that transfer energy to greater $|\boldsymbol{k}|$, the speedup in characteristic timescales that occurs as the cascade process

\footnotetext{
15 More accurately, on the sum and difference of these: the Elsasser potentials.
} 
continues (essentially as in the Kolmogorov picture) is almost entirely due to transfer to higher $k_{\perp}$, not higher $\kappa_{\|}^{\prime}$. In this sense, one may view that all parallel spectral transfer in RMHD is 'kinematic.'

Furthermore, since there is no parallel dissipation (see below), there is no drain at high $\kappa_{\|}^{\prime}$ to pull energy in that direction. The loss of energy moving towards higher $\kappa_{\|}^{\prime}$ is mainly due to the progressive transfer of energy towards higher $k_{\perp}$, which does ultimately lead to dissipation. This idea motivated Montgomery (1989) to argue that the reduced spectrum in the (global) parallel direction, $E\left(\kappa_{\|}^{\prime}\right)=\iint \mathrm{d} k_{x} \mathrm{~d} k_{y} E\left(k_{x}, k_{y}, \kappa_{\|}^{\prime}\right)$, will have an exponential 'survival probability' form (the integrand is the modal energy spectrum). Simulation results support the argument (Kinney \& McWilliams 1997; Oughton et al. 2004). An alternative suggestion, by Beresnyak (2015), is that the parallel spectrum calculated along field lines is really the Lagrangian frequency spectrum in disguise, and hence should be $\sim k_{\|}^{-2}$.

It is clear from the derivations outlined above that, in terms of the unscaled spatial coordinates, RMHD has $\nabla_{\perp} \gg \nabla_{\|}$, so that $\nabla^{2} \rightarrow \nabla_{\perp}^{2}$ when introducing simple scalar viscous or resistive dissipation. For these unscaled derivatives, this makes little difference because $\nabla^{2}=\nabla_{\perp}^{2}+\epsilon_{\mathrm{S}}^{2} \nabla_{\|}^{2}$, and although the parallel dissipation is present it is very small. However, when the rescaled coordinates are employed - as is often the case for numerical simulations - it is incorrect to include viscous dissipation in the form $\nu \nabla^{2} \boldsymbol{v}$ with a scalar $\nu$, because this, in effect, introduces a large viscosity acting on parallel gradients. While in certain approximations (e.g., Montgomery 1992) this might correspond to a real plasma physics effect, there is no basis for including it in a purely collisional MHD context. In the rescaled case, the dissipation operators in RMHD should involve $\nabla_{\perp}^{2}$, and not $\nabla^{2}$.

\subsection{RMHD with isotropic energy-containing scales?}

As the above derivations with $\delta b / B_{0} \ll 1$ have shown, for RMHD to be valid at all scales one requires also that the large scales be anisotropic with $\ell_{\perp} \ll \ell_{\|}$. Conversely, if $\mathrm{MHD}$ is (lengthscale) isotropic at energy-containing scales, still with $\delta b / B_{0} \ll 1$, then one has $\tau_{\mathrm{A}} \ll \tau_{\mathrm{NL}}$ for those scales and RMHD will not be a good approximation for them. Nonetheless, in such situations it is possible that perpendicular spectral transfer might quickly populate smaller scale modes for which RMHD is "locally" valid [meaning $\tau_{\mathrm{NL}}(\boldsymbol{k})<\tau_{\mathrm{A}}(\boldsymbol{k})$ ]. Indeed, it has been suggested that RMHD is a good approximation for inertial range fluctuations, even when the large scales do not obey the RMHD equations (e.g., Perez \& Boldyrev 2008; Beresnyak 2012). However it is unclear how to join together two such different models - consisting, say, of full MHD at the larger scales and RMHD at the smaller inertial range of scales.

If one considers full MHD with $\delta b / B_{0}=O(1)$ and spectral isotropy at the largest scales, then potential use of an RMHD approximation also encounters difficulty. On the plus side, the nonlinear and Alfvén timescales evaluated at the largest scales will not be grossly misordered, in contrast to the $\delta b / B_{0} \ll 1$ with $\ell_{\perp} \approx \ell_{\|}$case of the previous paragraph. However, almost always, parallel vari- ances will quickly be generated, and the RMHD restriction to the transverse (toroidal) modes will be violated (Dmitruk et al. 2005). In such circumstances, the details of which vary with plasma beta (Franci et al. 2015a,b; Oughton et al. 2016; Parashar et al. 2016), RMHD will not remain a good approximation to MHD for times much longer than a single large-scale nonlinear time.

What about using RMHD to describe sub-volumes of these $\delta b \approx B_{0}, \ell_{\perp} \approx \ell_{\|}$systems? For a small enough sub-volume, the local mean field $\langle\boldsymbol{B}\rangle_{\text {large, }}$ obtained by averaging over the large scales, could have a well-defined direction and also be strong, motivating use of RMHD in this region. However, $\langle\boldsymbol{B}\rangle_{\text {large }}$ will change in direction (and magnitude) on the global nonlinear timescale, $\tau_{\mathrm{NL}}$, presumably limiting the length of time such a model would be valid to $t \lesssim \tau_{\mathrm{NL}}$. There are also 'open boundary' issues associated with such an approach. First, the large eddies will sweep 'external' fluctuations into the sub-volume, and also sweep some 'original' fluctuations out of it. ${ }^{16}$ Second, Alfvén waves will propagate in and out of the region, typically in directions which differ from those of the sweeping. To account for these influences, some sort of boundary forcing would probably be needed. Collectively, these aspects limit the usefulness of such sub-volume models, perhaps to just statistical information.

\subsection{Perpendicular spectral transfer and phenomenology}

For RMHD, with anisotropic large-scale energycontaining eddies, and $\delta b / B_{0} \ll 1$, spectral transfer is dominantly in the perpendicular direction. Since $k_{\perp}>$ $k_{\|}$at all scales, the nonlinear timescale is mainly determined by the perpendicular wavenumber, i.e., $\tau_{\mathrm{NL}}(k) \approx$ $\tau_{\mathrm{NL}}\left(k_{\perp}\right)$. Furthermore, since nonlinear decorrelation is never slower than global Alfvénic decorrelation for this RMHD ordering, the Kolmogorov phenomenology is appropriate (cf. Matthaeus \& Zhou 1989; Zhou \& Matthaeus 2005) and one arrives at an inertial range spectral density (integrated over $k_{\|}$and azimuthal angle) of the form $E\left(k_{\perp}\right) \sim k_{\perp}^{-5 / 3}$. Note that for the purely 2D case, essentially the same argument follows, as discussed by Fyfe et al. (1977). A variant argument can be constructed in which the large-scale fluctuating magnetic field in the perpendicular plane becomes strong enough so that the perpendicular Alfvén time becomes smaller than $\tau_{\mathrm{NL}}\left(k_{\perp}\right)$. This may lead to a $k_{\perp}^{-3 / 2}$ Kraichnan spectral law instead of the Kolmogorov $-5 / 3$ behavior (Grappin et al. 1982, 1983).

\subsection{Relation to Critical Balance}

RMHD and phenomenologies based on the critical balance assumption (Goldreich \& Sridhar 1995), share a reliance on the equal timescale curve, $\tau_{\mathrm{NL}}(\boldsymbol{k}) \approx \tau_{\mathrm{A}}(\boldsymbol{k})$. However, this curve enters into these models in distinct ways, as we now discuss.

RMHD is a subset of compressible MHD that lacks all fast timescales, meaning those that approach zero as the applied magnetic field $B_{0}$ tends to infinity (e.g., M82). This requires that the dynamically active modes

\footnotetext{
16 To mitigate this effect, a Lagrangian RMHD model might be considered.
} 
have Alfvén timescales that remain of order the nonlinear timescale $\tau_{\mathrm{NL}}$, or larger, as $B_{0} \rightarrow \infty$. These restrictions lead to the condition expressed in Eq. (5), from which one readily arrives at the RMHD spectral anisotropy constraint, $k_{\|} \ll k_{\perp}$. Any mode $\boldsymbol{k}$ satisfying $\tau_{\mathrm{NL}}(\boldsymbol{k})<\tau_{A}(\boldsymbol{k})$ will clearly satisfy all the conditions for inclusion in the region of wavenumber space relevant to RMHD. The outer extent of this region is estimated by the equal timescale curve, $\tau_{\mathrm{NL}}(\boldsymbol{k}) \approx \tau_{\mathrm{A}}(\boldsymbol{k})$.

Intriguingly, this same (approximate) equality of the timescales is obtained through the assumptions leading to critical balance theory (Goldreich \& Sridhar 1995). Critical balance and RMHD share other properties as well, such as the dominance of perpendicular transfer and the lack of fluctuation variances parallel to $\boldsymbol{B}_{0}$. However, there are also fundamental differences between these models - notably in the dynamical scenarios leading to their respective reduced dynamical descriptions. Roughly speaking, RMHD emerges from the interaction of low (including zero) frequency Fourier modes, whereas critical balance is established via interaction of higher frequency Alfvén waves. Both chains of reasoning lead to the condition $\tau_{\mathrm{NL}}(\boldsymbol{k}) \lesssim \tau_{\mathrm{A}}(\boldsymbol{k})$, but in essence this is arrived at from the high frequency side in critical balance theory and from the low frequency side in RMHD. In addition, critical balance is usually interpreted more strongly, requiring (approximate) timescale equality, not just the above inequality.

We will not delve further into this and other technical aspects of the relationship here, except to emphasize that, while the equal timescale condition is conceptually relevant in both models, RMHD and critical balance are quite distinct. We will examine this relationship in greater detail in a forthcoming paper.

\section{DIMENSIONALITY QUESTIONS AND ISSUES}

As noted in the introduction, the extent to which RMHD encompasses the physics of 3D MHD and/or 2D MHD is an important question. Since RMHD is an approximation to the compressible 3D MHD equations, it is unlikely to have retained all of the physics of the original system. For example, compressible activity is always absent in RMHD, and typically so too are parallel fluctuations. Recall that RMHD is inherently incompressible while the $2 \mathrm{D}, 2.5 \mathrm{D}$, and $3 \mathrm{D}$ cases might be compressible or incompressible. Of the numerous comparisons that can be made between RMHD and either 2D or 3D MHD, some are more subtle than these examples. We have therefore found it useful to assemble a summary of whether or not various features are present, or relevant, in several guises of MHD (Table 1). Some of the listed features have already been discussed, some are obvious, and some are considered more fully below.

At least one issue is essentially semantic. RMHD has sometimes been described as "fully 3D"-presumably meaning that it depends on three spatial coordinates (e.g., Perez \& Boldyrev 2008; Beresnyak 2012; Mason et al. 2012; Howes 2015). However, such a description is somewhat misleading and might easily be misinterpreted. The key issue is that the parallel coordinate which appears in the RMHD equations and their solutions, $s^{\prime}=\epsilon_{\mathrm{S}} z$, is necessarily large-scale. This is because of the spectral anisotropy required to eliminate high-frequency Alfvén waves in a system with a strong mean field, as is evident in the RMHD derivations. Thus, it is more accurate to say RMHD has a restricted $3 \mathrm{D}$ dependence, or, that RMHD is $3 \mathrm{D}$ but with the parallel coordinate restricted to be large-scale. In addition, "fully 3D" might suggest to the unwary reader that all functional degrees of freedom are present, but, as noted in $\S 3.3$, poloidal polarization is exactly absent in RMHD.

Another category of comparisons is that of conservation laws. In this regard, RMHD in some sense falls between (incompressible) 2D/2.5D MHD and 3D MHD. In each of these systems the total fluctuation energy (per unit mass), $E=\left\langle|\boldsymbol{v}|^{2}\right\rangle / 2+\left\langle|\boldsymbol{b}|^{2}\right\rangle / 2$ and the cross helicity, $H_{c}=\langle\boldsymbol{v} \cdot \boldsymbol{b}\rangle$, are conserved. (The brackets denote an ensemble or volume average with appropriate boundary conditions.) For $3 \mathrm{D}$ MHD with $B_{0}=0$, the fluctuation magnetic helicity $H_{m}=\langle\boldsymbol{a} \cdot \boldsymbol{b}\rangle$ is also an invariant; here $\boldsymbol{b}=\nabla \times \boldsymbol{a}$ with $\boldsymbol{a}$ the vector potential for the magnetic fluctuations. When $B_{0} \neq 0, H_{m}$ is no longer an invariant so that one might suspect this would also be the case in RMHD. However, in the usual RMHD with $v_{z}=b_{z}=0$, one has $\boldsymbol{a}=a_{z} \hat{\boldsymbol{z}}$ and thus $H_{m}=0$. In a trivial sense this is also a conserved quantity, although clearly not a very useful one. One can define a total magnetic helicity, $H_{m}^{\text {tot }}=\langle\boldsymbol{a} \cdot \boldsymbol{B}+\ldots\rangle=\left\langle a_{z} B_{0}+\ldots\right\rangle$, based on $\boldsymbol{B}=\boldsymbol{B}_{0}+\boldsymbol{b}$ (Montgomery 1981). In the common situation where $B_{0}$ is uniform, this becomes $B_{0}\left\langle a_{z}\right\rangle$, essentially just the average of $a_{z}$. The number of (quadratic) invariants and their positive definiteness (or not) are important determinants of the Gibbsian 'absolute equilibrium' statistical mechanics of the non-dissipative systems, and of associated expectations for cascade behavior in dissipative cases (Kraichnan 1973; Montgomery 1981; Stribling \& Matthaeus 1990).

Features that rely on strict two-dimensionality will not be present in RMHD. For example, in 2D MHD, there is ideal conservation of mean-square vector potential $\left\langle a_{z}^{2}\right\rangle$, and an antidynamo theorem (Pouquet 1978), but these do not hold in RMHD. It is interesting that conservation of $\left\langle a_{z}^{2}\right\rangle$ in $2 \mathrm{D}$ appears to be related to the presence of so-called $1 / f$ noise (Dmitruk et al. 2011); issues related to this in RMHD are discussed in $\$ 5.1$.

Similarly, aspects that require full three-dimensionality are not likely to be present in RMHD. For example, a mean field with fluctuations that are strictly transverse means that the following are all absent in RMHD: magnetic loops, magnetic nulls, null interchange reconnection, and reversals of magnetic field lines. Their absence may limit the relevance of RMHD studies of the acceleration and transport of energetic particles. On the other hand the Jokipii theorem (Jokipii et al. 1993; Jones et al. 1998) that constrains transport in systems with an ignorable coordinate does not pertain to RMHD, and in this way it is a more realistic model than strictly $2 \mathrm{D}$ turbulence (Shalchi \& Hussein 2014).

Spectral transfer in RMHD is also peculiar, being neither strictly 2D nor fully 3D. RMHD transfer is dominantly in the perpendicular planes, with much weaker transfer in the $\boldsymbol{B}_{0}$ direction (Kinney \& McWilliams 1998; Oughton et al. 2004; Dmitruk et al. 2005). In this, it is very similar to 3D MHD with strong, or even moderate, mean field (e.g., Montgomery \& Turner 1981; Shebalin et al. 1983; Oughton et al. 1994; Matthaeus et al. 1996; Goldreich \& Sridhar 1995; Maron \& Goldreich 2001; Cho 
Table 2

Some ways in which RMHD is 'like' and 'not like' 2D MHD and 3D MHD.

\begin{tabular}{|c|c|c|}
\hline & $2 \mathrm{D}$ & $3 \mathrm{D}$ \\
\hline Like & $\begin{array}{l}\text { Transverse } \\
\text { Low frequency (no high } \omega=\boldsymbol{k} \cdot \boldsymbol{V}_{A} \text { modes) } \\
\text { Dominated by perp spectral transfer }\end{array}$ & $\begin{array}{l}\text { No ignorable coordinate, so avoids issue of Jokipii et al. (1993) theorem } \\
\text { No 2D antidynamo theorem (Pouquet 1978) } \\
\text { Some parallel spectral transfer } \\
\left.\text { Propagating Alfvén waves, at low frequencies (with } k_{\perp} \neq 0\right) \text {. }\end{array}$ \\
\hline $\begin{array}{l}\text { Not } \\
\text { Like }\end{array}$ & $\begin{array}{l}\left\langle a_{z}^{2}\right\rangle \text { not conserved } \\
\text { No antidynamo theorem } \\
\text { No ignorable coordinate } \\
\text { Not exactly "zero frequency" } \\
\text { Some parallel spectral transfer }\end{array}$ & $\begin{array}{l}\text { Strict transversality of RMHD fluctuations mandates } \\
\text { - no reversals, no loops, no magnetic nulls, no null interchange } \\
\left.\text { - no magnetic helicity (aside from the "cheat" helicity, }\left\langle a_{z} B_{0}\right\rangle\right) \text {. } \\
\text { Lengthscale inequality } \nabla_{\|} \ll \nabla_{\perp} \text { and spectral anisotropy mean } \\
\text { - no parallel dissipation } \\
\text { - no parallel shocks } \\
\text { - no slab waves } \\
\text { - lack of power at high } k_{\|} \text {: problematic for energetic particle scattering } \\
\text { - often large-scale structures cannot be expected to be highly } \\
\text { anisotropic in this sense }\end{array}$ \\
\hline
\end{tabular}

\& Vishniac 2000; Cho et al. 2002; Cho \& Lazarian 2003; Müller et al. 2003; Boldyrev 2006; Bigot et al. 2008; Grappin \& Müller 2010; Alexakis 2011; Mason et al. 2012). Of course in the $3 \mathrm{D}$ case there are poloidal field couplings that can contribute to parallel transfer, and even if they are weak in 3D, they are completely absent in RMHD. The approximate two-dimensionality of RMHD spectral transfer may be considered, crudely, as both "like 2D MHD" and "like 3D MHD."

Table 2 presents, in list form, a summary of ways in which RMHD is like, and also not like, both 2D and 3D MHD.

\subsection{Quasi-invariance and $1 / f$ noise}

As a particular example of the RMHD model's intermediate nature, we document here the presence of a $1 / f$ signal in long-time driven RMHD. Previously Dmitruk et al. (2011) have investigated the emergence of $1 / f$ noise at low frequencies in fluid systems that have more than one quadratic invariant (Kraichnan \& Montgomery 1980). Such systems can exhibit inverse cascade behavior when driven at intermediate length scales. As stated above, 3D incompressible MHD with a mean DC magnetic field $B_{0}$ (and no cross helicity) admits only one quadratic invariant, the energy. However for strong $B_{0}$ and when driven, this system becomes anisotropic and begins to two-dimensionalize. The mean-square magnetic potential $\left\langle a_{z}^{2}\right\rangle$ never becomes fully conserved, but is "quasi-conserved" in random epochs, sometimes for hundreds of characteristic times. In this scenario, $1 / f$ noise appears without a second strict ideal conservation law. We also find this property in RMHD, as we document in Figures 1 and 2. As RMHD is a special case of the strong mean field limit of 3D MHD this is perhaps not surprising.

For this demonstration, a periodic Fourier spectral method RMHD code was employed with resolution of $256^{2} \times 32$ and mean field $B_{0}=8$. A forced and dissipative simulation was carried out for 1000 large-scale nonlinear times, with forcing in the (low) wavenumber modes $1<|\boldsymbol{k}|<4$. A range of $1 / f$ behavior can be seen in the Eulerian frequency spectrum for $b_{x}\left(\boldsymbol{x}_{0}, t\right)$, where $\boldsymbol{x}_{0}$ is an arbitrary fixed position (Fig. 1). This appears at frequencies between $f \approx 0.1$ and $f \approx 0.001$, extending to frequencies much lower than the reciprocal of the large-scale eddy turnover time, $f_{c}=1 / \tau_{\mathrm{NL}} \approx 1$. This

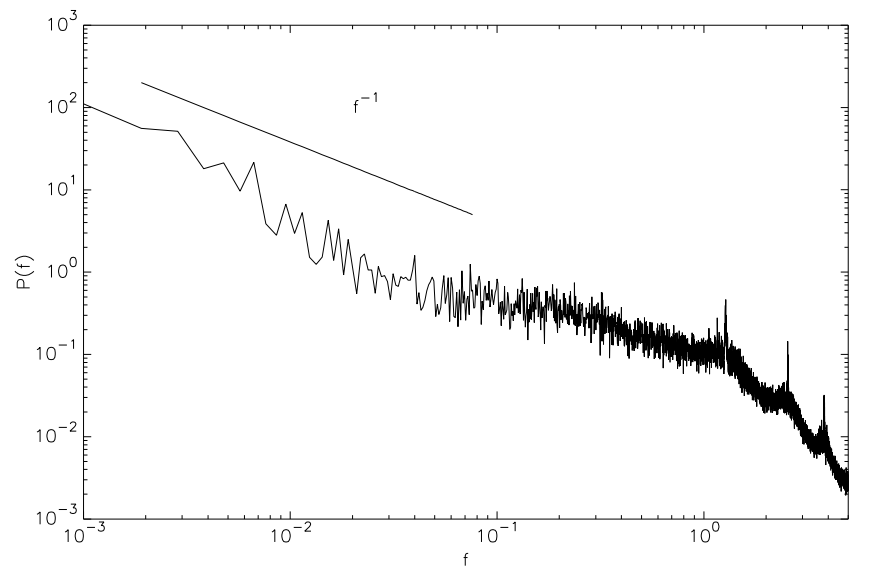

Figure 1. Eulerian frequency spectrum from a forced RMHD simulation. The approximate $1 / f$ spectrum is found in a frequency range much lower than the reciprocal of the large-scale eddy turnover time $f_{c}=1 / \tau_{\mathrm{NL}} \approx 1$. In this low frequency range, there is a great excess of power relative to a system that becomes uncorrelated at frequencies $<f_{c}$.

is the same kind of low-frequency behavior seen in other systems that are of the inverse cascade type or of the quasi-invariant type (Dmitruk et al. 2011). ${ }^{17}$

Two familiar features of $1 / f$ systems can be seen in Figure 2: (i) that the time series show long periods of nearly stationary behavior punctuated by level changes; and (ii) that much of the energy condenses into a small subset of modes. In the RMHD case, the condensed modes are the $2 \mathrm{D}$ ones $\left(\kappa_{\|}^{\prime}=0\right)$ with the shortest perpendicular wavenumbers, i.e., $\boldsymbol{k}_{\perp}=(1,0)$ and $(0,1)$. Further physical interpretation of these properties may be found in the references (Dmitruk et al. 2011).

\section{ENERGY WAVE EQUATION}

RMHD contains an interesting dynamical feature that is not present in either 2D, 2.5D, or 3D MHD. Consider a coordinate space plane $s^{\prime}=$ const. that is oriented per-

\footnotetext{
17 We note that, as usual, at very low frequencies it is difficult to obtain precise powerlaw behavior because of the enormous simulation time needed to achieve statistical convergence. However the important point is that in the " $1 / f$ " range there is far more power at very low frequencies than would be expected for a system that became stationary and uncorrelated at frequencies much lower than the reciprocal large-scale turnover time.
} 


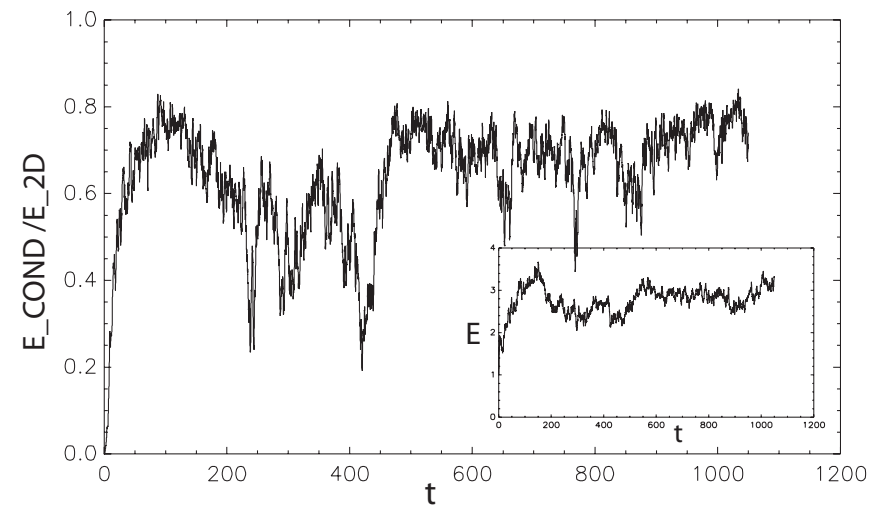

Figure 2. Energy (kinetic plus magnetic) in condensed modes as a fraction of the energy in the 2D modes, vs. time. In this run, the $2 \mathrm{D}$ energy varies in time as $15-65 \%$ of the total energy. Time is in units of nonlinear time evaluated for total energy at the longest wavelength modes. Inset: total energy vs. time. Data is from the same simulation as Fig. 1.

pendicular to the mean magnetic field $B_{0}$. Denote the Elsasser energies in each such plane as

$$
W^{ \pm}\left(s^{\prime}, t\right)=\frac{1}{2} \int\left|\boldsymbol{z}^{ \pm}\left(x, y, s^{\prime}, t\right)\right|^{2} \mathrm{~d} x \mathrm{~d} y .
$$

Remarkably, these physically interesting quantities obey linear wave equations, even when strong nonlinear turbulence is present. To obtain this result, dot $z^{ \pm}$with Eqs. (9) and then sum over the perpendicular coordinates, with appropriate transverse boundary conditions (Dmitruk et al. 2001). Ignoring dissipative effects yields

$$
\left(\frac{\partial}{\partial t} \mp V_{A}^{\prime \prime} \frac{\partial}{\partial s^{\prime}}\right) W^{ \pm}\left(s^{\prime}, t\right)=0 .
$$

A similar equation is obtained by van Ballegooijen et al. (2011, Appendix C), when considering a variant of RMHD applied to inhomogeneous flux tubes.

An example illustrating this linear wave behavior is shown in Fig. 3, which uses data from a (periodic) spectral method simulation of the ideal RMHD equations. The initial state is a (finite) Fourier series approximation to all the energy being in the single plane $s^{\prime}=100$. Energy and cross helicity are well-conserved throughout the run. The lefthand panel is a surface plot of $W^{-}\left(s^{\prime}, t\right)$ for several crossing times of the periodic domain. Finite numerical resolution ${ }^{18}$ leads to some jitter in the amplitudes of $W^{ \pm}$, but the overall invariance of the planar energies is evident. Figure 4 is a still from an animation included with the online version of this paper, showing the time evolution of the $W^{ \pm}\left(s^{\prime}, t\right)$ and the vector potential $a(x, y)$ and vorticity $\omega(x, y)$ in the planes where $W^{ \pm}$ have their largest values. $W^{+}$and $W^{-}$are seen to propagate through each other multiple times with only minor (numerical) distortion. Moreover, one can also see that although the propagating energies $W^{ \pm}$are (essentially) constant, the nonlinear dynamics within the transverse planes can be active and strong without violating the linearity of the wave equation.

Why does this wave equation for the 'plane energies' holds for RMHD, but not for 3D MHD? The key feature

\footnotetext{
18 Specifically, the proper representation of a $\delta\left(s^{\prime}\right)$ function requires infinitely many Fourier modes.
}
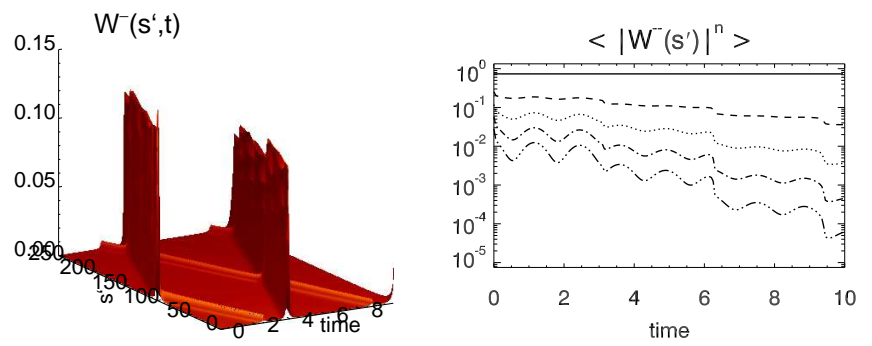

Figure 3. Left: surface plot of $W^{-}\left(s^{\prime}, t\right)$ showing its wave behavior. Right: averages over $s^{\prime}$ of $\left|W^{-}\left(s^{\prime}, t\right)\right|^{n}$ for $n=1, \ldots, 5$. Data is from an ideal $(\nu=\eta=0)$ RMHD spectral simulation with resolution $128^{2} \times 256$. Results for $W^{+}$are similar.
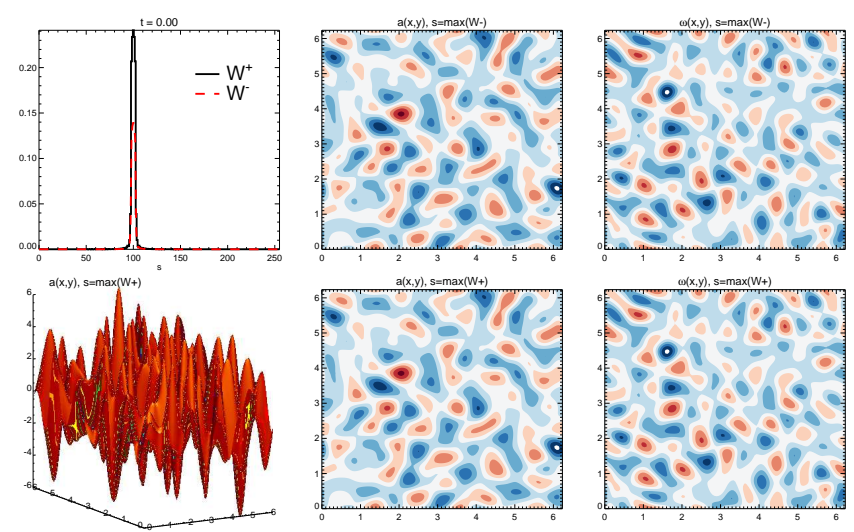

Figure 4. Initial frame from the mpeg movie available in the online version. This shows the time evolution of $W^{ \pm}\left(s^{\prime}, t\right)$ and the vector potential $a$ and vorticity $\omega$ in the $s^{\prime}$ planes with maximum $W^{ \pm}$.

is the transverse nature of the fluctuations: For incompressible 3D MHD, the RHS of Eq. (22) is not zero but rather

$$
-\frac{\partial}{\partial z} \int \mathrm{d} x \mathrm{~d} y\left(z_{\|}^{+} p^{*}+z_{\|}^{-} \frac{z_{+}^{2}}{2}\right),
$$

where $s^{\prime}$ has been replaced by $z$ since the parallel coordinate is not rescaled in 3D MHD. Similarly, on the LHS, $V_{A}^{\prime \prime} \partial_{s^{\prime}} \rightarrow V_{A}^{3 \mathrm{D}} \partial_{z}$. Here, $p^{*}$ is the fluid plus magnetic pressure (see $\S 3$ ).

There are several ways that Eq. (23) can be zero or small:

1. It is exactly zero for strictly transverse fluctuations.

2. It is $\approx 0$ if the parallel amplitudes are small compared to the transverse ones.

3. It is $\approx 0$ if $\partial_{z}=O(\epsilon)$ and $V_{A}^{3 \mathrm{D}}=O(1 / \epsilon)$, for some $\epsilon \ll 1$. The $V_{A}^{3 \mathrm{D}}$ ordering is needed so that the $V_{A}^{3 \mathrm{D}} \partial_{z}$ wave term in Eq. (22) remains $O(1)$. Clearly, this is closely related to RMHD.

Regarding the first case, we note that in both incompressible and compressible 3D MHD, parallel fluctuations are generated immediately even if they are not present initially, although the level can be rather low depending upon the plasma beta and other parameters (Oughton et al. 2016). Thus, the first case is unlikely to be stable, 
but may transition quickly to the second, small parallel amplitudes, case.

Focusing again on the case in which the linear wave equation for $W^{ \pm}\left(s^{\prime}, t\right)$, Eq. (22), does hold, we emphasize the following point regarding the generality and applicability of the RMHD model. The (summed) wave energy in toroidal modes is conserved at every value of the parallel coordinate $s^{\prime}$ for the continuum ideal RMHD model. This implies an infinite number of pointwise invariant quantities in RMHD - all of which are absent in the 3D MHD model. So although RMHD is a reduced model that necessarily lacks properties of the full MHD framework, it nonetheless also possesses this infinite class of invariants that are irrelevant in MHD, except perhaps as quasi-invariants in special circumstances.

Strongly related to this class of invariants, is the fact that $\left\langle\left|W^{ \pm}\left(s^{\prime}, t\right)\right|^{n}\right\rangle$ are continuum ideal invariants for all integers $n \geq 1$, where $\langle\cdots\rangle$ indicates averaging over $s^{\prime}$. This is readily shown using Eq. (22). For numerical solutions, however, spatial discretization ${ }^{19}$ means that only the $n=1$ case remains an invariant for the numerical method, since the other cases involve quantities that are of higher order than quadratic (Ghosh et al. 1993). This is clear in the righthand panel of Fig. 3, which shows that $\left\langle W^{-}\right\rangle$is very well conserved, but for $n>1$ the $\left\langle\left|W^{-}\right|^{n}\right\rangle$ are increasingly less well conserved.

\section{CONCLUSIONS}

We have presented a discussion of the assumptions and approximations involved in RMHD, clarifying the distinction between those that are primary or fundamental and those that are consequences. Points of stress have been the subtle aspects in the different derivations and the relationship among the several available approaches to these derivations. Our discussion of the structure of the RMHD equations is intentionally detailed, although we would not claim it is exhaustive. An emphasis has been a consideration of the complex relationship that exists between RMHD and other MHD models, mainly 2D MHD and 3D MHD, for both the incompressible and compressible cases. There are similarities and dissimilarities relative to each of these models. On balance, we would conclude that RMHD is neither $2 \mathrm{D}$ not $3 \mathrm{D}$, although it is unambiguously incompressible. Guidance for proper application of RMHD may be found in the details of the derivations, and also by examining the desirability, in a specific application, of adopting in RMHD a model that resembles $2 \mathrm{D}$ and $3 \mathrm{D}$, and differs from them in the many ways listed in Tables 1 and 2 and discussed in $§ 5$. Application of RMHD beyond the asymptotic parameters range for which it can be derived should be done with caution, as its special properties (and perhaps in particular the infinite class of ideal wave energy invariants) may cause RMHD solutions to depart from full MHD solutions in important ways.

\section{APPENDIX}

\section{REVIEW OF KP AND S76 DERIVATIONS}

Section 2.2 gave rather terse overviews of the two classic derivations of RMHD (KP, S76). Here we outline these in more detail.

19 Or equivalently in the case of spectral methods, the finite number of Fourier modes employed.
Kadomtsev and Pogutse (KP) presented the classical derivation for tokamak plasmas. It begins with the assumptions that the mean magnetic field $B_{0} \hat{z}$ is much stronger than the fluctuations transverse to it, $\boldsymbol{b}_{\perp}$, and that the mechanical pressure is negligible compared to magnetic pressure, i.e., the plasma beta, $\beta_{\mathrm{p}}$, is formally zero. Everything else is ordered relative to the associated small parameter,

$$
\epsilon_{\mathrm{KP}}=b_{\perp} / B_{0} \ll 1,
$$

where $b_{\perp}$ is the rms amplitude of the transverse magnetic fluctuation. The derivation specializes to the fluctuations of greatest interest, which are those that vary slowly along the mean field direction $\hat{z}$, and for which the plasma motion is purely transverse to $\hat{\boldsymbol{z}}$. Correspondingly, for any fluctuation field, one takes its parallel spatial derivative $\nabla_{z}$ to be suppressed by a factor $\epsilon_{\mathrm{KP}}$ relative to the $\nabla_{\perp}$ gradients, while also assuming that the parallel velocity fluctuation $v_{z} \sim \epsilon_{\mathrm{KP}} v_{\perp}$ is down by the same factor relative to the perpendicular velocity $v_{\perp}$.

An additional assumption is made immediately, that the parallel magnetic fluctuation is small $b_{z} \ll b_{\perp}$; specifically $b_{z} \sim \epsilon_{\mathrm{KP}}^{2} B_{0}$. From the momentum equation, using the smallness of $\nabla_{z}$ and $b_{z} / B_{0}$, one shows that the assumption that $v_{z}=0$ at leading order is consistent, because there are no forces in that direction. One then proceeds to estimate the divergence of the fluctuation velocity $\boldsymbol{v}$ from the magnetic induction equation, finding that $\nabla \cdot \boldsymbol{v}=O\left(\epsilon_{\mathrm{KP}}^{2}\right)$. [Or $O\left(\epsilon_{\mathrm{KP}}^{3}\right)$ if it transpires that the time derivatives are $O\left(\epsilon_{\mathrm{KP}}\right)$. Although KP do not explicitly note that $\partial_{t}$ scales this way, it is consistent with their results.] Furthermore $\nabla_{\perp} \cdot \boldsymbol{b}_{\perp}=O\left(\epsilon_{\mathrm{KP}}^{2}\right)$. One concludes that at leading order the motion is incompressible and transverse to $\boldsymbol{B}_{0}$, and that $\boldsymbol{v}_{\perp}=\nabla_{\perp} \phi(x, y, z) \times \hat{\boldsymbol{z}}$ and $\boldsymbol{b}_{\perp}=\nabla_{\perp} A(x, y, z) \times \hat{\boldsymbol{z}}$ vary slowly in the parallel direction and may be determined from the scalar potentials $\phi$ and $A$ respectively. (Because $\beta_{\mathrm{p}}=0$ in this derivation, $b_{z}$ plays the role of an incompressible pressure: the perpendicular divergence of the equation of motion yields a Poisson equation for $b_{z}$.)

Strauss derivation. A well-known and closely related derivation also given in the context of tokamak plasmas is due to Strauss (S76). The starting point is the small inverse aspect ratio of the toroidal plasma (minor radius/major radius). With the mean magnetic field in the axial direction $\hat{\boldsymbol{z}}$, it is argued that the characteristic scales in the perpendicular and axial directions are also in this ratio. Denoting these as $\ell_{\perp}$, and $\ell_{\|}$, respectively, we have a distinct small parameter

$$
\epsilon_{\mathrm{S}}=\ell_{\perp} / \ell_{\|} \ll 1,
$$

and it this that is employed as the expansion parameter for the S76 derivation, rather than $\epsilon_{\mathrm{KP}}$.

S76 postulates an ordering in which $\nabla_{\perp}=O(1)$, while $\nabla_{\|}=O\left(\epsilon_{\mathrm{S}}\right), b_{x}, b_{y}=O\left(\epsilon_{\mathrm{S}}\right)$, with $\stackrel{B_{z}}{=}=B_{0}+b_{z}$, $B_{0}=O(1)$ and $b_{z}=O\left(\epsilon_{\mathrm{S}}^{2}\right)$, all of which are equivalent to assumptions made by KP. Time derivatives are assumed to be $O\left(\epsilon_{\mathrm{S}}\right)$. The plasma beta is assumed small (whereas KP set $\beta_{\mathrm{p}}=0$ ) so that there is a fluctuating pressure, taken to be $p=O\left(\epsilon_{\mathrm{S}}^{2}\right)$. A series of arguments is then developed that lead quickly to the RMHD equations, (1)-(2).

First, due to the slowly varying parallel derivative, it 
is found that the transverse magnetic field is solenoidal up to $O\left(\epsilon_{\mathrm{S}}^{3}\right)$. Next, examining the momentum equation (as in the KP derivation), one finds a vanishing parallel force, so that $v_{z}=0$ at the leading order. But upon inserting this result into the equation for the advancement of the magnetic vector potential one finds that $\boldsymbol{v}=\nabla \phi(x, y, z) \times \hat{\boldsymbol{z}}$ and thus the velocity fluctuation lies in planes perpendicular to the mean field, and the flow is incompressible. The assumption of an initial uniform constant density then makes this property valid at all times. Having established the forms of the magnetic and velocity fluctuations - both of which are transverse to $\hat{\boldsymbol{z}}$, solenoidal in the perpendicular planes, and slowly varying in the $z$ direction - one readily arrives at the form of the RMHD equations given in Eqs. (1) and (2).

The approximations and arguments given in the KP and S76 derivations are similar. In particular, they both adopt orderings that eliminate magnetosonic modes, thus rendering the full MHD system at least temporarily devoid of fast timescale compressible motions. This underlies the importance of attaining an incompressible state as a precursor to arriving at RMHD equations. The KP and S76 approaches also contain similar ambiguities. For example, in neither case is the relationship between $\epsilon_{\mathrm{KP}}$ and $\epsilon_{\mathrm{S}}$ emphasized. In effect, each work assumes that $\epsilon_{\mathrm{S}}=\epsilon_{\mathrm{KP}}$ [cf. Appendix A of Oughton et al. (2004)].

\section{REFERENCES}

Alexakis, A. 2011, Phys. Rev. E, doi: 10.1103/PhysRevE. 84.056330, 84, 056330

Batchelor, G. K. 1970, The Theory of Homogeneous Turbulence (Cambridge, UK: Cambridge University Press)

Beresnyak, A. 2012, Mon. Not. R. Astron. Soc., doi $10.1111 /$ j.1365-2966.2012.20859.x, 422, 3495

. 2015, Astrophys. J. Lett., doi: 10.1088/2041-8205/801/1/L9, 801, L9

Bhattacharjee, A., Ng, C. S., \& Spangler, S. R. 1998, Astrophys. J., 494, 409

Bigot, B., Galtier, S., \& Politano, H. 2008, Phys. Rev. E, doi: 10.1103/PhysRevE.78.066301, 78, 066301

Boldyrev, S. 2006, Phys. Rev. Lett., doi:

10.1103/PhysRevLett.96.115002, 96, 115002

Bondeson, A. 1985, Phys. Fluids, doi: 10.1063/1.865246, 28, 2406

Cho, J., \& Lazarian, A. 2003, Mon. Not. R. Astron. Soc., doi: 10.1046/j.1365-8711.2003.06941.x, 345, 325

Cho, J., Lazarian, A., \& Vishniac, E. T. 2002, Astrophys. J., doi: $10.1086 / 324186,564,291$

Cho, J., \& Vishniac, E. T. 2000, Astrophys. J., doi: 10.1086/309213, 539, 273

Craya, A. 1958, Publ. Sci. Tech. Ministère de l'Air, 345

Dahlburg, J. P., Montgomery, D., \& Matthaeus, W. H. 1985, J. Plasma Phys., 34, 1

Dalena, S., Rappazzo, A. F., Dmitruk, P., Greco, A., \& Matthaeus, W. H. 2014, Astrophys. J., doi: 10.1088/0004-637X/783/2/143, 783, 143

Dmitruk, P., \& Gómez, D. O. 1997, Astrophys. J., doi: $10.1086 / 310760,484, \mathrm{~L} 83$

Dmitruk, P., Gómez, D. O., \& DeLuca, E. E. 1998, Astrophys. J., doi: 10.1086/306182, 505, 974

Dmitruk, P., Gómez, D. O., \& Matthaeus, W. H. 2003, Phys. Plasmas, doi: 10.1063/1.1602698, 10, 3584

Dmitruk, P., Matthaeus, W. H., Milano, L. J., \& Oughton, S. 2001, Phys. Plasmas, doi: 10.1063/1.1344563, 8, 2377

Dmitruk, P., Matthaeus, W. H., \& Oughton, S. 2005, Phys. Plasmas, doi: 10.1063/1.2128573, 12,112304

Dmitruk, P., Mininni, P. D., Pouquet, A., Servidio, S., \& Matthaeus, W. H. 2011, Phys. Rev. E, doi: 10.1103/PhysRevE.83.066318, 83, 066318

Einaudi, G., Velli, M., Politano, H., \& Pouquet, A. 1996, Astrophys. J., doi: 10.1086/309893, 457, L113

Franci, L., Landi, S., Matteini, L., Verdini, A., \& Hellinger, P. 2015a, Astrophys. J., doi: 10.1088/0004-637X/812/1/21, 812, 21 Franci, L., Verdini, A., Matteini, L., Landi, S., \& Hellinger, P. 2015b, Astrophys. J. Lett., doi: 10.1088/2041-8205/804/2/L39, 804, L39
Fyfe, D., Montgomery, D., \& Joyce, G. 1977, J. Plasma Phys., 17, 369

Ghosh, S., Hossain, M., \& Matthaeus, W. H. 1993, Comp. Phys. Comm., doi: 10.1016/0010-4655(93)90103-J, 74, 18

Goldreich, P., \& Sridhar, S. 1995, Astrophys. J., doi: 10.1086/175121, 438, 763

Grappin, R. 1986, Phys. Fluids, doi: 10.1063/1.865536, 29, 2433

Grappin, R., Frisch, U., Léorat, J., \& Pouquet, A. 1982, Astron. Astrophys., 105, 6

Grappin, R., \& Müller, W.-C. 2010, Phys. Rev. E, doi: 10.1103/PhysRevE.82.026406, 82, 026406

Grappin, R., Pouquet, A., \& Léorat, J. 1983, Astron. Astrophys. 126,51

Herring, J. R. 1974, Phys. Fluids, doi: 10.1063/1.1694822, 17, 859

Howes, G. G. 2015, J. Plasma Phys., doi: 10.1017/S0022377814001056, 81, 325810203

Jokipii, J. R., Kota, J., \& Giacalone, J. 1993, Geophys. Res. Lett., 20, 1759

Jones, F., Jokipii, J. R., \& Baring, M. 1998, Astrophys. J., 509, 238

Kadomtsev, B. B., \& Pogutse, O. P. 1974, Sov. Phys.-JETP, 38, 283, [Zh. Eksp. Teor. Fiz. 65, 575 (1973)]

Kinney, R., \& McWilliams, J. C. 1997, J. Plasma Phys., doi: $10.1017 / \mathrm{S} 0022377896005284,57,73$

-. 1998, Phys. Rev. E, doi: 10.1103/PhysRevE.57.7111, 57, 7111

Klainerman, S., \& Majda, A. 1982, Comm. Pure Appl. Math. doi: 10.1002 cpa.3160350503, 35, 629

Kraichnan, R. H. 1973, J. Fluid Mech., 59, 745

Kraichnan, R. H., \& Montgomery, D. C. 1980, Rept. Prog. Phys. 43,547

Krause, F., \& Rädler, K.-H. 1980, Mean-Field Magnetohydrodynamics and Dynamo Theory (Berlin: Akademie-Verlag)

Kreiss, H.-O. 1980, Comm. Pure Appl. Math., doi: 10.1002/cpa.3160330310, 33, 399

Kruger, S. E., Hegna, C. C., \& Callen, J. D. 1998, Phys. Plasmas, doi: $10.1063 / 1.873152,5,4169$

Kubo, R. 1963, J. Math. Phys., 4, 174

Maron, J., \& Goldreich, P. 2001, Astrophys. J., doi: $10.1086 / 321413,554,1175$

Mason, J., Perez, J. C., Boldyrev, S., \& Cattaneo, F. 2012, Phys. Plasmas, doi: 10.1063/1.3694123, 19, 055902

Matthaeus, W. H., \& Brown, M. R. 1988, Phys. Fluids, doi $10.1063 / 1.866880,31,3634$

Matthaeus, W. H., Ghosh, S., Oughton, S., \& Roberts, D. A. 1996, J. Geophys. Res., doi: 10.1029/95JA03830, 101, 7619

Matthaeus, W. H., \& Zhou, Y. 1989, Phys. Fluids B, doi: $10.1063 / 1.859110,1,1929$

Milano, L. J., Dmitruk, P., Mandrini, C. H., \& Gómez, D. O. 1999, Astrophys. J., 521, 889

Montgomery, D. 1981, in Presented at the US-Japan Workshop on 3D MHD Studies for Toroidal Devices, Oak Ridge, Tenn., 19 Oct. 1981, ed. D. D. Schnack

Montgomery, D. C. 1982, Phys. Scr., doi: 10.1088/0031-8949/1982/T2A/009, T2/1, 83

_ 1989, Lecture Notes on Turbulence (Singapore: World Scientific), 75, lecture Notes from the NCAR-GTP Summer School, June 1987

-. 1992, J. Geophys. Res., 97, 4309

Montgomery, D. C., \& Turner, L. 1981, Phys. Fluids, doi: 10.1063/1.863455, 24, 825

Morrison, P. J., \& Hazeltine, R. P. 1984, Phys. Fluids, 27, 886

Müller, W.-C., Biskamp, D., \& Grappin, R. 2003, Phys. Rev. E, doi: 10.1103/PhysRevE.67.066302, 67, 066302

Oughton, S., Dmitruk, P., \& Matthaeus, W. H. 2004, Phys. Plasmas, doi: 10.1063/1.1705652,11,2214

Oughton, S., Matthaeus, W. H., Dmitruk, P., et al. 2001, Astrophys. J., doi: 10.1086/320069, 551, 565

Oughton, S., Matthaeus, W. H., Wan, M., \& Parashar, T. N. 2016, J. Geophys. Res., doi: 10.1002/2016JA022496, 121, 5041

Oughton, S., Priest, E. R., \& Matthaeus, W. H. 1994, J. Fluid Mech., doi: 10.1017/S0022112094002867, 280, 95

Parashar, T. N., Matthaeus, W. H., Wan, M., \& Oughton, S. 2016. Astrophys. J., doi: 10.3847/0004-637X/824/1/44, 824, 44

Perez, J. C., \& Boldyrev, S. 2008, Astrophys. J., doi: 10.1086/526342,672, L61

Pouquet, A. 1978, J. Fluid Mech., doi 10.1017/S0022112078001950, 88, 1

Rappazzo, A. F., Matthaeus, W. H., Ruffolo, D., Servidio, S., \& Velli, M. 2012, Astrophys. J. Lett., doi: 10.1088/2041-8205/758/1/L14, 758, L14

Rappazzo, A. F., Velli, M., \& Einaudi, G. 2010, Astrophys. J., doi: $10.1088 / 0004-637 \mathrm{X} / 722 / 1 / 65,722,65$

—. 2013, Astrophys. J., doi: 10.1088/0004-637X/771/2/76, 771 , 76 
Rappazzo, A. F., Velli, M., Einaudi, G., \& Dahlburg, R. B. 2008, Astrophys. J., doi: 10.1086/528786, 677, 1348

Schekochihin, A. A., Cowley, S. C., Dorland, W., et al. 2009, Astrophys. J. Suppl. Ser, doi: 10.1088/0067-0049/182/1/310, 182,310

Scheper, R. A., \& Hassam, A. B. 1999, Astrophys. J., doi: $10.1086 / 306702,511,976$

Schmidtmann, O., Feudel, F., \& Seehafer, N. 1998, J. Phys. A, doi: $10.1088 / 0305-4470 / 31 / 34 / 016,31(34), 7141$

Servidio, S., Matthaeus, W. H., Wan, M., et al. 2014, Astrophys. J., doi: 10.1088/0004-637X/785/1/56, 785, 56

Shalchi, A., \& Hussein, M. 2014, Astrophys. J., doi: $10.1088 / 0004-637 \mathrm{X} / 794 / 1 / 56,794,56$

Shebalin, J. V., Matthaeus, W. H., \& Montgomery, D. 1983, J. Plasma Phys., doi: 10.1017/S0022377800000933, 29, 525
Snodin, A. P., Ruffolo, D., Oughton, S., Servidio, S., \& Matthaeus, W. H. 2013, Astrophys. J., doi: 10.1088/0004-637X/779/1/56, 779, 56

Strauss, H. R. 1976, Phys. Fluids, doi: 10.1063/1.861310, 19, 134

—. 1977, Phys. Fluids, doi: 10.1063/1.862018, 20, 1354

Stribling, T., \& Matthaeus, W. H. 1990, Phys. Fluids B, 2, 1979 van Ballegooijen, A. A., Asgari-Targhi, M., Cranmer, S. R., \& DeLuca, E. E. 2011, Astrophys. J., doi:

10.1088/0004-637X/736/1/3,736, 3

Zank, G. P. \& Matthaeus, W. H. 1992, J. Plasma Phys., doi: 10.1017/S002237780001638X, 48, 85

- 1993, Phys. Fluids A, doi: 10.1063/1.858780, 5, 257

Zhou, Y., \& Matthaeus, W. H. 2005, Phys. Plasmas, doi: $10.1063 / 1.1887187,12,056503$ 